\title{
Aveiro Discretization Method in Mathematics: A New Discretization Principle*
}

\author{
L.P. Castro, H. Fujiwara, M.M. Rodrigues, S. Saitoh and V.K. Tuan
}

\begin{abstract}
We found a very general discretization method for solving wide classes of mathematical problems by applying the theory of reproducing kernels. An illustration of the generality of the method is here performed by considering several distinct classes of problems to which the method is applied. In fact, one of the advantages of the present method - in comparison to other well-known and well established methods - is its global nature and no need of special or very particular data conditions. Numerical experiments have been made, and consequent results are here exhibited. Due to the powerful results which arise from the application of the present method, we consider that this method has everything to become one of the next generation methods of solving general analytical problems by using computers. In particular, we would like to point out that we will be able to solve very global linear partial differential equations satisfying very general boundary conditions or initial values (and in a somehow independent way of the boundary and domain). Furthermore, we will be able to give an ultimate sampling theory and an ultimate realization of the consequent general reproducing kernel Hilbert spaces. The general theory is here presented in a constructive way, and containing some related historical and concrete examples.
\end{abstract}

\footnotetext{
* Accepted author's manuscript (AAM) published in [L.P. Castro, H. Fujiwara, M.M. Rodrigues, S. Saitoh, V.K. Tuan: Aveiro Discretization Method in Mathematics: A New Discretization Principle, Panos Pardalos and Themistocles M. Rassias (edts), Mathematics without Boundaries: Surveys in Pure Mathematics, 37-92, Springer-Verlag, New York, 2014.] [DOI: 10.1007/978-14939-1106-6_3] The final publication is available at Springer via http://dx.doi.org/10.1007/978-14939-1106-6_3

L. P. Castro, M.M. Rodrigues and S. Saitoh

CIDMA-Center for Research and Development in Mathematics and Applications, Department of Mathematics, University of Aveiro, 3810-193 Aveiro, Portugal, e-mail: castro@ua.pt; mrodrigues@ua.pt; saburou.saitoh@gmail.com

H. Fujiwara

Graduate School of Informatics, Kyoto University, Kyoto, 606-8501, Japan, e-mail: fujiwara@acs.i.kyoto-u.ac.jp

V.K. Tuan

Department of Mathematics, University of West Georgia, 1601 Maple Street, Carrollton, GA 30118, USA, e-mail: vu@westga.edu
} 


\section{Introduction}

Nowadays, the importance and influence of reproduction kernel Hilbert spaces is identified in several different subareas of mathematics and their applications. In some cases, such importance is increased when convenient modifications and applications of different techniques are additionally implemented. A major ingredient which gave already rise to important and global results was simply the consideration of linear mappings within the framework of Hilbert spaces in a reproduction kernels identification process. This gave already rise to a very powerful and also natural use of reproducing kernels in the framework of Hilbert spaces (as it may be seen in [35]) which, in its turn, had several very significant consequences during the last decades.

As a somehow natural consequence of the research project proposed by S. Saitoh in the University of Aveiro some years ago, and being developed until now, it was introduced a new line of research within the Functional Analysis and Applications Group of this university (involving not only researchers from this university but also collaborators from abroad). Among some other fruitful consequences of that project within reproduction kernel Hilbert spaces, in here we would like to present the so-called Aveiro Discretization Method in Mathematics. Interestingly enough, this name was identified by S. Saitoh in the night just before one of his talks presented in the "9th International Conference on Mathematical Problems in Engineering, Aerospace and Science" (Vienna, Austria, 10-14 July 2012). In such a talk, it was presented by the first time (in an oral and non detailed way) the main ideas of the method that we are now presenting in here in a complete form.

As main ingredients for the starting points of the method, we may identify the general theory of reproducing kernels, the Tikhonov regularization procedure, consequent approximate solutions for bounded linear operator equations on Hilbert spaces, and optimal numerical solutions.

In particular, as examples of possible applications, we will be able to solve very general linear partial differential equations (PDEs) satisfying very general boundary conditions and initial values - independently of the boundary and domains. Furthermore, we will be able to give clearly an ultimate sampling theory and ultimate realizations of general reproducing kernel Hilbert spaces. We would like to develop the general theory in a self contained manner with some related history and many concrete examples.

The remaining part of this work is organized in the following way. In Section 2, we establish a general formula giving an optimal inverse by using a finite number of output data in the framework of Hilbert spaces. In Section 3, we shall establish the convergence property of our approximate inverses in Section 2. In order to show our history for some fundamental inverses with the typical example of the Laplace transform, we shall refer to several typical inverses in Sections 3-9 and in Section 10, we shall give our final and new approximate inversion of the Laplace transform. In Section 11, we shall introduce the Paley-Wiener spaces and Sobolev spaces (due to the necessity of these spaces in our new discretization principle). In Section 12, we shall derive results for general linear ordinary differential equations, and in Section 13 we derive concrete results for various typical partial differential equations. In 
Sections 14 and 15, we will introduce results for convolution and singular integral equations, respectively. In Section 16, we shall give several numerical experiments, and we end up with a general discussion in Section 17 (mostly from the viewpoint of numerical analysis).

\section{Principal Formulation of the Inverse by Using a Finite Number of Data}

We shall firstly establish our fundamental inversion formula by using a finite number of data in the framework of Hilbert spaces. In view of this, following [35, 40], we shall start by introducing a general theory for linear mappings in the framework of Hilbert spaces.

Let $\mathscr{H}$ be a Hilbert (possibly finite-dimensional) space, and consider $E$ to be an abstract set and $\mathbf{h}$ a Hilbert $\mathscr{H}$-valued function on $E$. Then, we are able to consider the linear transform

$$
f(p)=(\mathbf{f}, \mathbf{h}(p))_{\mathscr{H}}, \quad \mathbf{f} \in \mathscr{H},
$$

from $\mathscr{H}$ into the linear space $\mathscr{F}(E)$ comprising all the complex valued functions on $E$. In order to investigate the linear mapping (1), we form a positive definite quadratic form function $K(p, q)$ on $E \times E$ defined by

$$
K(p, q)=(\mathbf{h}(q), \mathbf{h}(p))_{\mathscr{H}} \quad \text { on } \quad E \times E .
$$

Then, we obtain the following fundamental result.

\section{Proposition 1.}

(I) The range of the linear mapping (1) by $\mathscr{H}$ is characterized as the reproducing kernel Hilbert space $H_{K}(E)$ admitting the reproducing kernel $K(p, q)$ whose characterization is given by the two properties: $(i) K(\cdot, q) \in H_{K}(E)$ for any $q \in E$ and, (ii) for any $f \in H_{K}(E)$ and for any $p \in E,(f(\cdot), K(\cdot p))_{H_{K}(E)}=f(p)$.

(II) In general, we have the inequality

$$
\|f\|_{H_{K}(E)} \leq\|\mathbf{f}\|_{\mathscr{H}}
$$

Here, for any member $f$ of $H_{K}(E)$ there exists a uniquely determined $\mathbf{f}^{*} \in \mathscr{H}$ satisfying

$$
f(p)=\left(\mathbf{f}^{*}, \mathbf{h}(p)\right)_{\mathscr{H}} \quad \text { on } \quad E
$$

and

$$
\|f\|_{H_{K}(E)}=\left\|\mathbf{f}^{*}\right\|_{\mathscr{H}}
$$

(III) In general, we have the inversion formula in (1) in the form

$$
f \mapsto \mathbf{f}^{*}
$$

in (II) by using the reproducing kernel Hilbert space $H_{K}(E)$. 
The typical ill-posed problem (1) will become a well-posed problem, because the image space of (1) is characterized as the reproducing kernel Hilbert space $H_{K}(E)$ with the isometric identity (3), which may be considered as a generalization of the Pythagorean theorem.

However, this viewpoint is a mathematical one and is not a numerical one, as we shall refer to later.

We shall consider the inversion in (4) formally. However, this idea will be very important for the general inversions and for our new discretization method.

Following the above general situation, we shall recall the general theory in the following general situation (cf. also $[34,35]$ ): Let $\left\{\mathbf{v}_{j}\right\}$ be a complete orthonormal basis for $\mathscr{H}$. Then, for

$$
v_{j}(p)=\left(\mathbf{v}_{j}, \mathbf{h}(\mathbf{p})\right)_{\mathscr{H}},
$$

we have

$$
\mathbf{h}(\mathbf{p})=\sum_{j}\left(\mathbf{h}(\mathbf{p}), \mathbf{v}_{j}\right) \mathscr{H} \mathbf{v}_{j}=\sum_{j} \overline{v_{j}(p)} \mathbf{v}_{j}
$$

Hence, by setting

$$
\overline{\mathbf{h}}(p)=\sum_{j} v_{j}(p) \mathbf{v}_{j}
$$

we may write

$$
\overline{\mathbf{h}}(\cdot)=\sum_{j} v_{j}(\cdot) \mathbf{v}_{j}
$$

Thus, we shall define

$$
(f, \overline{\mathbf{h}}(p))_{H_{K}}=\sum_{j}\left(f, v_{j}\right)_{H_{K}} \mathbf{v}_{j}
$$

For simplicity, we will write as follows:

$$
H_{K}=H_{K}(E),
$$

here and in the sequel. Then, we have the following result.

Proposition 2. We assume that for $f \in H_{K}$, it holds

$$
(f, \overline{\mathbf{h}})_{H_{K}} \in \mathscr{H}
$$

and for all $p \in E$,

$$
\left(f,(\mathbf{h}(p), \mathbf{h}(\cdot))_{\mathscr{H}}\right)_{H_{K}}=\left((f, \overline{\mathbf{h}})_{H_{K}}, \mathbf{h}(p)\right)_{\mathscr{H}}
$$

Then, we have

$$
\|f\|_{H_{K}} \leqq\left\|(f, \overline{\mathbf{h}})_{H_{K}}\right\|_{\mathscr{H}} \text {. }
$$

If $\{\mathbf{h}(p) ; p \in E\}$ is complete in $\mathscr{H}$, then the equality takes place in (5).

Furthermore, we assume:

$$
\left(\mathbf{f}_{0},(f, \overline{\mathbf{h}})_{H_{K}}\right)_{\mathscr{H}}=\left(\left(\mathbf{f}_{0}, \mathbf{h}\right)_{\mathscr{H}}, f\right)_{H_{K}} \quad \text { for } \quad \mathbf{f}_{0} \in N(L) .
$$


Then, we have, for $\mathbf{f}^{*}$ in (II) and (III),

$$
\mathbf{f}^{*}=(f, \overline{\mathbf{h}})_{H_{K}} .
$$

In particular, note that the basic assumption $(f, \overline{\mathbf{h}})_{H_{K}} \in \mathscr{H}$ in Proposition 2, is, in general, not valid for many analytical problems and we need some delicate treatment for the inversion (see [35]). In view of this, let us analyse again the possibilities for the linear mapping defined by (1). In order to derive a general inversion formula for (1) that is widely applicable in analysis, we shall assume that both Hilbert spaces $\mathscr{H}$ and $H_{K}$ are given as $\mathscr{H}=L_{2}(T, d m), H_{K} \subset L_{2}(E, d \mu)$, on the sets $T$ and $E$, respectively. Note that for $d m, d \mu$ measurable sets $T, E$ we assume that they are the Hilbert spaces comprising $d m, d \mu-L_{2}$ integrable complex-valued functions, respectively. Therefore, we shall consider the integral transform

$$
f(p)=\int_{T} F(t) \overline{h(t, p)} d m(t) .
$$

Here, $h(t, p)$ is a function on $T \times E, h(\cdot, p) \in L_{2}(T, d m)$, and $F \in L_{2}(T, d m)$. The corresponding reproducing kernel for (2) is given by

$$
K(p, q)=\int_{T} h(t, q) \overline{h(t, p)} d m(t) \quad \text { on } \quad E \times E .
$$

The norm of the reproducing kernel Hilbert space $H_{K}$ is represented as $L_{2}(E, d \mu)$. Under these situations, we obtain the following result.

Proposition 3. We assume that an approximating sequence $\left\{E_{N}\right\}_{N=1}^{\infty}$ of E satisfies

(a) $E_{1} \subset E_{2} \subset \cdots \subset E_{n} \subset \cdots$,

(b) $\bigcup_{N=1}^{\infty} E_{N}=E$,

(c) $\int_{E_{N}} K(p, p) d \mu(p)<\infty, \quad N=1,2, \ldots$

Then, for $f \in H_{K}$ satisfying $\int_{E_{N}} f(p) h(t, p) d \mu(p) \in L_{2}(T, d m)$ for any $N$, the sequence

$$
\left\{\int_{E_{N}} f(p) h(t, p) d \mu(p)\right\}_{N=1}^{\infty}
$$

converges to $F^{*}$ in (4) in Proposition 1 in the sense of the $L_{2}(T, d m)$ norm.

Practically, for many cases, the assumptions in Proposition 3 will be satisfied automatically, and so Proposition 3 may be applied in all those cases. To construct the inversion formula is - in general - difficult. However, the formulation in Proposition 3 may be considered as a natural one. Indeed, this may be realized if we recognize that it was given as the strong convergence in the Hilbert space $L_{2}(T, d m)$.

Proposition 3 will give a new viewpoint and method for the fundamental Fredholm integral equation of the first kind (6). Our method and solution will have the following properties:

(P1) We use the naturally determined reproducing kernel Hilbert space $H_{K}$ which is determined by the integral kernel. 
(P2) The solution is given in the sense of $\mathscr{H}$ norm convergence.

(P3) Our solution (inverse) is given by $f^{*}$ in Proposition 1.

(P4) For the ill-posed problem in (1), our method gives a well-posed solution.

In general, in a linear problem, when we cannot guarantee the eventual uniqueness of a solution continuously dependent on the data, then the linear problem is called an ill-posed problem in the Hadamard sense. The linear problem (1) or (6) has been considered a typical ill-posed problem. Our method gives a characterization of the image; that is, the solution space. For the inverse or solution, we consider the minimum norm one (among many solutions) and then we have the isometric relation in Proposition 1; that is, our solution has uniqueness and continuity properties. However, this is a mathematical and theoretical viewpoint. In practical and physical linear systems, the observation data will be a finite number of data containing error or noises, and so we will meet various delicate numerical problems and there exists a field of ill-posed problems. In order to overcome such cases, we examined several methods, as we shall state in the sequel.

Our new idea is based on the approximate realization of the abstract Hilbert space $H_{K}$ by taking a finite number of points of $E$, because, in general, the reproducing kernel Hilbert space $H_{K}$ has a complicated structure. Therefore, we will implement the following procedure: By taking a finite number of points $\left\{p_{j}\right\}_{j=1}^{n}$, we set

$$
K\left(p_{j}, p_{j^{\prime}}\right):=a_{j j^{\prime}}
$$

Consequently, if the matrix $A:=\left\|a_{j j^{\prime}}\right\|$ is positive definite, then, the corresponding norm in $H_{A}$ comprising the vectors $\mathbf{x}=\left(x_{1}, x_{2}, \ldots, x_{n}\right)$ is determined by

$$
\|\mathbf{x}\|_{H_{A}}^{2}=\mathbf{x}^{*} \widetilde{A} \mathbf{x}
$$

where $\widetilde{A}=\overline{A^{-1}}=\left\|\widetilde{a_{j j^{\prime}}}\right\|$ (see [35], p. 250). This property may be confirmed directly in a non difficult way.

When we approximate the reproducing kernel Hilbert space $H_{K}$ by the vector space $H_{A}$, then from Proposition 2, the following theorem is directly derived.

Theorem 1. In the linear mapping

$$
f(p)=(\mathbf{f}, \mathbf{h}(p))_{\mathscr{C}}, \quad \mathbf{f} \in \mathscr{H}
$$

for

$$
A_{n}=\left\{p_{1}, p_{2}, \ldots, p_{n}\right\},
$$

the minimum norm inverse $\mathbf{f}_{A_{n}}^{*}$ satisfying

$$
f\left(p_{j}\right)=\left(\mathbf{f}, \mathbf{h}\left(p_{j}\right)\right)_{\mathscr{H}}, \quad \mathbf{f} \in \mathscr{H}
$$

is given by

$$
\mathbf{f}_{A_{n}}^{*}=\sum_{j} \sum_{j^{\prime}} f\left(p_{j}\right) \widetilde{a_{j j^{\prime}}} \mathbf{h}\left(p_{j^{\prime}}\right)
$$


where $\widetilde{a_{j j^{\prime}}}$ are assumed the elements of the complex conjugate inverse of the positive definite Hermitian matrix $A_{n}$ constituted by the elements

$$
a_{j j^{\prime}}=\left(\mathbf{h}\left(p_{j^{\prime}}\right), \mathbf{h}\left(p_{j}\right)\right)_{\mathscr{H}} .
$$

Here, the positive definiteness of $A_{n}$ is a basic assumption.

\section{Convergence of the Approximate Inverses}

The following theorem deals with the convergence of our approximate inverses in Theorem 1. The case of the Laplace transform was proved in [44].

Theorem 2. Let $\left\{p_{j}\right\}_{j=1}^{\infty}$ be a sequence of distinct points on $E$, that is the positive definiteness in Theorem 1 for any $n$ and a uniqueness set for the reproducing kernel Hilbert space $H_{K}$; that is, for any $f \in H_{K}$, if all $f\left(p_{j}\right)=0$, then $f \equiv 0$. Then, in the space $\mathscr{H}$, it holds

$$
\lim _{n \rightarrow \infty} \mathbf{f}_{A_{n}}^{*}=\mathbf{f}^{*}
$$

Proof. By Theorem 1, we obtain the monotonicity

$$
\left\|\mathbf{f}_{A_{n}}^{*}\right\|_{\mathscr{H}} \leq\left\|\mathbf{f}_{A_{m}}^{*}\right\|_{\mathscr{H}}, \quad \text { if } \quad 0<n<m,
$$

and

$$
\left\|\mathbf{f}_{A_{n}}^{*}\right\|_{\mathscr{H}} \leq\left\|\mathbf{f}^{*}\right\|_{\mathscr{H}}, \quad \text { if } \quad n>0 .
$$

The sequence $\left\{\mathbf{f}_{A_{n}}^{*}\right\}$ is uniformly bounded in norm by $\left\|\mathbf{f}^{*}\right\|_{\mathscr{H}}$ in the Hilbert space $\mathscr{H}$, therefore, there exists a subsequence $\left\{\mathbf{f}_{A_{n_{j}}}^{*}\right\}$ that converges weakly to a member $\mathbf{f}^{* *} \in \mathscr{H}$ and $\left\|\mathbf{f}^{* *}\right\|_{\mathscr{H}} \leq \liminf _{j \rightarrow \infty}\left\|\mathbf{f}_{A_{n_{j}}}^{*}\right\|_{\mathscr{H}}$. Then, we have

$$
\lim _{j \rightarrow \infty} f_{A_{n_{j}}}^{*}(p):=\lim _{j \rightarrow \infty}\left(\mathbf{f}_{A_{n_{j}}}^{*}, \mathbf{h}(p)\right)_{\mathscr{H}}=: f^{*}(p) \in H_{K} .
$$

In particular, since $f_{A_{n_{j}}}^{*}\left(p_{k}\right)=f\left(p_{k}\right)$ when $n_{j} \geq k$, we have

$$
f^{*}\left(p_{k}\right)=\lim _{j \rightarrow \infty} f_{A_{n_{j}}}^{*}\left(p_{k}\right)=f\left(p_{k}\right), \quad k>0 .
$$

From the uniqueness assumption, $f^{*}(z)=f(z)$. Therefore, $\mathbf{f}^{* *}=\mathbf{f}^{*}$. hence,

The sequence $\left\{\left\|\mathbf{f}_{A_{n_{j}}}^{*}\right\| \mathscr{H}\right\}$ is monotone increasing, and bounded above by $\left\|\mathbf{f}^{*}\right\|_{\mathscr{H}}$,

$$
\left\|\mathbf{f}^{*}\right\|_{\mathscr{H}} \leq \liminf _{j \rightarrow \infty}\left\|\mathbf{f}_{A_{n_{j}}^{*}}\right\|_{\mathscr{H}} \leq\left\|\mathbf{f}^{*}\right\|_{\mathscr{H}}
$$

Consequently,

$$
\lim _{j \rightarrow \infty}\left\|\mathbf{f}_{A_{n_{j}}}^{*}\right\|_{\mathscr{H}}=\left\|\mathbf{f}^{*}\right\|_{\mathscr{H}}
$$


The subsequence $\left\{\mathbf{f}_{A_{n_{j}}}^{*}\right\}$ converges weakly to $\mathbf{f}^{*}$, and the sequence of their norms $\left\{\left\|\mathbf{f}_{A_{n_{j}}}^{*}\right\|_{\mathscr{H}}\right\}$ converges to $\left\|\mathbf{f}^{*}\right\|_{\mathscr{H}}$. Therefore, the subsequence $\left\{\mathbf{f}_{A_{n_{j}}}^{*}\right\}$ converges strongly to $\mathbf{f}^{*}$. We claim now that the whole sequence $\left\{\mathbf{f}_{A_{n}}^{*}\right\}$ converges strongly to $\mathbf{f}^{*}$. If not, there would exist an $\varepsilon>0$ and a subsequence $\left\{\mathbf{f}_{A_{n_{l}}^{*}}^{*}\right\}$ such that

$$
\left\|\mathbf{f}^{*}-\mathbf{f}_{A_{n_{l}}}^{*}\right\|_{\mathscr{H}} \geq \varepsilon, \quad l>0
$$

By the same techniques as above, from the sequence $\left\{\mathbf{f}_{A_{n_{l}}}^{*}\right\}$ one can choose a subsequence convergent strongly to $\mathbf{f}^{*}$, that is a contradiction. Hence, $\mathbf{f}_{A_{n}}^{*}$ converges in norm to $\mathbf{f}^{*}$.

The result is a surprisingly simple and pleasant one. Indeed, we can obtain directly the ultimate realization of the reproducing kernel Hilbert spaces and the ultimate sampling theory that are much more simpler than the known derivations (cf. [35], pp. 92-96).

Theorem 3 (Ultimate realization of reproducing kernel Hilbert spaces). In our general situation and for a uniqueness set $\left\{p_{j}\right\}$ of the set $E$ satisfying the linearly independence in Theorem 1, we obtain

$$
\|f\|_{H_{K}}^{2}=\left\|\mathbf{f}^{*}\right\|_{\mathscr{H}}^{2}=\lim _{n \rightarrow \infty} \sum_{j} \sum_{j^{\prime}} f\left(p_{j}\right) \widetilde{a_{j j^{\prime}}} \overline{f\left(p_{j^{\prime}}\right)} .
$$

Theorem 4 (Ultimate sampling theory). In our general situation and for a uniqueness set $\left\{p_{j}\right\}$ of the set $E$ satisfying the linearly independence in Theorem 1, we obtain

$$
\begin{aligned}
f(p) & =\lim _{n \rightarrow \infty}\left(\mathbf{f}_{A_{n}}^{*}, \mathbf{h}(p)\right)_{\mathscr{H}} \\
& =\lim _{n \rightarrow \infty}\left(\sum_{j} \sum_{j^{\prime}} f\left(p_{j}\right) \widetilde{a_{j j^{\prime}}} \mathbf{h}\left(p_{j^{\prime}}\right), \mathbf{h}(p)\right)_{\mathscr{H}} \\
& =\lim _{n \rightarrow \infty} \sum_{j} \sum_{j^{\prime}} f\left(p_{j}\right) \widetilde{a_{j j^{\prime}}} K\left(p, p_{j^{\prime}}\right) .
\end{aligned}
$$

In Theorem 1, Theorem 2, Theorem 3 and Theorem 4 there are several relevant observations. Namely, for any given finite number of $f\left(p_{j}\right), j=1,2, \ldots, n$, the result in Theorem 1 is valid. Meanwhile, Theorem 2 and Theorem 3 are valid when we consider the sequence $f\left(p_{j}\right), j=1,2, \ldots$, for any member $f$ of $H_{K}$. The sequence $f\left(p_{j}\right), j=1,2, \ldots$, for any member $f$ of $H_{K}$ is characterized by the convergence of (13) in Theorem 3. Then, any member $f$ of $H_{K}$ is represented by (14) in terms of the sequence $f\left(p_{j}\right), j=1,2, \ldots$, in Theorem 4 .

Here, we shall consider the following problem: In our general setting in Proposition 1 , we assume that we observed the values $f\left(p_{j}\right)=\alpha_{j}, j=1,2, \ldots, n$, for a finite number of points $\left\{p_{j}\right\}$. Then, for the whole value $f(p)$ of the set $E$, how can we consider it? 
One idea is to consider the function $f_{1}(p)$ : among the functions satisfying the conditions $f\left(p_{j}\right)=\alpha_{j}, j=1,2, \ldots, n$, we consider the minimum norm member $f_{1}(p)$ in $H_{K}(E)$. This function $f_{1}(p)$ is determined by the following formula (as we see easily)

$$
f_{1}(p)=\sum_{j=1}^{n} C_{j} K\left(p, p_{j}\right)
$$

where, the constants $\left\{C_{j}\right\}$ are determined by the formula:

$$
\sum_{j=1}^{n} C_{j} K\left(p_{j^{\prime}}, p_{j}\right)=\alpha_{j^{\prime}}, \quad j^{\prime}=1,2, \ldots, n
$$

(being clear that we assume that $\left\|K\left(p_{j^{\prime}}, p_{j}\right)\right\|$ is positive definite).

Meanwhile, by Theorem 1 , we can consider the function $f_{2}(p)$ defined by

$$
f_{2}(p)=\left(\mathbf{f}_{A_{n}}^{*}, \mathbf{h}(p)\right)_{\mathscr{H}}
$$

in terms of $\mathbf{f}_{A_{n}}^{*}$ in Theorem 1. This interpolation formula is depending on the linear system.

We shall show our short history for our various inverse formulas by the typical real inversion of the Laplace transform

$$
(L f)(p)=\int_{0}^{\infty} e^{-t p} F(t) d t
$$

and our first main aim is to obtain a consequent real inversion formula following the new idea.

\section{On the Known Real Inversion Formulas of the Laplace Transform}

In order to see what is known about the situation on the real inversion formula of the Laplace transform, we shall first recall the well-known inversion formulas. Here we denote by $f$ the Laplace transform of $F ; f=L F$.

The most popular formulas may be the following:

$$
\begin{aligned}
& \lim _{n \rightarrow \infty} \frac{(-1)^{n}}{n !}\left(\frac{n}{t}\right)^{n+1} f^{(n)}\left(\frac{n}{t}\right)=F(t), \\
& \lim _{n \rightarrow \infty} \prod_{k=1}^{n}\left(1+\frac{t}{k} \frac{d}{d t}\right) \frac{n}{t} f\left(\frac{n}{t}\right)=F(t) .
\end{aligned}
$$

For (16), we refer to the books by Post [31] and Widder [46], and for (17) we refer to the book by Widder [46]. 
We obtained the analytical real inversion formula for the Laplace transform after long steps within the method and reasoning of Section 2 (see also [35]):

$$
f(p)=\int_{0}^{\infty} e^{-p t} F(t) d t
$$

for $p>0$, where $F: \mathbb{R}^{+} \rightarrow \mathbb{C}$ is a measurable function satisfying

$$
\int_{0}^{\infty}|F(t)|^{2} d t<\infty
$$

For the polynomial of degree $2 N+2$,

$$
\begin{aligned}
P_{N}(\xi):= & \sum_{0 \leq v \leq n \leq N} \frac{(-1)^{v+1}(2 n) ! \xi^{n+v}}{(n+1) !(n-v) !(n+v) ! v !} \\
& \cdot\left\{\frac{(2 n+1) \xi^{2}}{n+v+1}-\left(\frac{2 n+1}{n+v+1}+3 n+1\right) \xi+n(n+v+1)\right\},
\end{aligned}
$$

we set

$$
F_{N}(t)=\int_{0}^{\infty} f(p) e^{-p t} P_{N}(p t) d p
$$

Then, we have

$$
\lim _{N \rightarrow \infty} \int_{0}^{\infty}\left|F(t)-F_{N}(t)\right|^{2} d t=0 .
$$

Furthermore, the estimation of the truncation error of $F_{N}(t)$ was also given in [1,37].

We recall also the formula by [32, p. 221]: For the Laplace transform

$$
\int_{0}^{b} e^{-p t} F(t) d t=f(p)
$$

we have

$$
\begin{aligned}
F(t)= & \left.\frac{2 t b^{-1}}{\pi} \frac{d}{d u} \int_{0}^{u} \frac{G(v)}{(u-v)^{\frac{1}{2}}} d v\right|_{u=t^{2} b^{-2}} ; \\
G(v)= & v^{-\frac{1}{2}} \frac{2}{\pi} \int_{0}^{\infty} d y \cos \left(y \cosh ^{-1} v^{-1}\right) \cosh \pi y \\
& \cdot \int_{0}^{\infty} d z \cos (z y)(\cosh z)^{-\frac{1}{2}} \int_{0}^{\infty} d p f(p) J_{0}\left(p \frac{b}{(\cosh z)^{\frac{1}{2}}}\right)
\end{aligned}
$$

where $J_{0}$ denotes the Bessel function. Unfortunately, in this very complicated formula, the characteristic properties on both functions $F$ and $f$ such that the inversion formula holds true were not given.

Peng and Chung (cf. [29]) gave the formula:

$$
F(t)=\lim _{\sigma \rightarrow \infty} \bar{F}_{\sigma}(t), \quad \bar{F}_{\sigma}(t)=\sum_{j=1}^{\infty} \frac{(-1)^{j-1}}{(j-1) !} e^{j \sigma} \frac{\sigma}{t} f\left(\frac{j \sigma}{t}\right) .
$$


Additionally, Tuan and Duc ([43]) gave:

$$
F(t)=\lim _{n \rightarrow \infty} n \sum_{j=1}^{\infty} \frac{(-1)^{j-1}}{(j-1) !} e^{n j t} f(n j) .
$$

Furthermore, we also refer to the related articles $[35,36]$. The problem may also be related to analytic extension problems; cf. [24, 25, 36]. In addition, in direct connection with Laplace transform inversion problems, we also would like to refer to $[3,4,14,20,26,30,45]$.

For the above typical difficult problem, we proceeded in the following ways.

\section{Best Approximations}

Let $L$ be any bounded linear operator from a reproducing kernel Hilbert space $H_{K}$ into a Hilbert space $\mathscr{H}$. Then, the following problem is a classical and fundamental problem known as the best approximate mean square norm problem. For any member $\mathbf{d}$ of $\mathscr{H}$, we would like to find

$$
\inf _{f \in H_{K}}\|L f-\mathbf{d}\|_{\mathscr{H}}
$$

It is clear that we are considering operator equations, generalized solutions and corresponding generalized inverses within the framework of $f \in H_{K}$ and $\mathbf{d} \in \mathscr{H}$, having in mind

$$
L f=\mathbf{d} \text {. }
$$

However, this problem has a complicated structure, specially in the infinite dimension Hilbert spaces case, leading in fact to the consideration of generalized inverses (in the Moore-Penrose sense). Following our theory, we can realize its complicated structure. Anyway, the problem turns to be well-posed within the reproducing kernels theory framework in the following proposition.

Proposition 4. For any member $\mathbf{d}$ of $\mathscr{H}$, there exists a function $\tilde{f}$ in $H_{K}$ satisfying

$$
\inf _{f \in H_{K}}\|L f-\mathbf{d}\|_{\mathscr{H}}=\|L \tilde{f}-\mathbf{d}\|_{\mathscr{H}}
$$

if and only if, for the reproducing kernel Hilbert space $H_{k}$ admitting the kernel defined by $k(p, q)=\left(L^{*} L K(\cdot, q), L^{*} L K(\cdot, p)\right)_{H_{K}}$,

$$
L^{*} \mathbf{d} \in H_{k}
$$

Furthermore, when there exists a function $\tilde{f}$ satisfying (24), there exists a uniquely determined function that minimizes the norms in $H_{K}$ among the functions satisfying the equality, and its function $f_{\mathbf{d}}$ is represented as follows:

$$
f_{\mathbf{d}}(p)=\left(L^{*} \mathbf{d}, L^{*} L K(\cdot, p)\right)_{H_{k}} \quad \text { on } \quad E .
$$


Here, the adjoint operator $L^{*}$ of $L$, as we see, from

$$
\left(L^{*} \mathbf{d}\right)(p)=\left(L^{*} \mathbf{d}, K(\cdot, p)\right)_{H_{K}}=(\mathbf{d}, L K(\cdot, p))_{\mathscr{H}}
$$

is represented by known $\mathbf{d}, L, K(p, q)$, and $\mathscr{H}$. From this proposition, we see that the problem is well formulated by the theory of reproducing kernels (i.e., the existence, uniqueness and representation of the solutions are well formulated). In particular, note that the adjoint operator is represented in a good way; this fact will be very important in our framework. The extremal function $f_{\mathbf{d}}$ is the Moore-Penrose generalized inverse $L^{\dagger} \mathbf{d}$ of the equation $L f=\mathbf{d}$. The criteria (25) is involved and so the Moore-Penrose generalized inverse $f_{\mathbf{d}}$ is not good, when the data contain error or noises in some practical cases. We shall see this complicated structure by using the Laplace transform in the next section.

\section{The Laplace Transform by Moore-Penrose Generalized Inverses}

Here, we will consider the (modified) Laplace transform

$$
(L f)(p):=p(\mathscr{L} f)(p)=p \int_{0}^{\infty} e^{-t p} f(t) d t .
$$

Let us fist fix some notation. The $\operatorname{ABS}[0, \infty)$ denotes the set of all real-valued absolutely continuous functions on $[0, \infty)$ (and we recall that we are using $\mathbb{R}^{+}$for denoting the half-line $(0, \infty))$. By a "weight" we simply mean a continuous function $w: \mathbb{R}^{+} \rightarrow \mathbb{R}^{+}$. Given a weight $w$, we define

$$
\begin{aligned}
L_{2}(w) & :=\left\{f: \mathbb{R}^{+} \rightarrow \mathbb{R}: f \text { is measurable and }\|f\|_{L_{2}(w)}<\infty\right\}, \\
H_{K}(w) & :=\left\{f \in \operatorname{ABS}[0, \infty): f(0)=0,\|f\|_{H_{K}(w)}<\infty\right\}
\end{aligned}
$$

where the norms are given by

$$
\|f\|_{L_{2}(w)}:=\left(\int_{0}^{\infty}|f(p)|^{2} w(p) d p\right)^{\frac{1}{2}}, \quad\|f\|_{H_{K}(w)}:=\left(\int_{0}^{\infty}\left|f^{\prime}(t)\right|^{2} w(t) d t\right)^{\frac{1}{2}}
$$

respectively.

Let us now recall some fundamental results.

Proposition 5 ([41]). Assume that for a given pair $w$ and $u$ of weights,

$$
M:=\iint_{\mathbb{R}^{+} \times \mathbb{R}^{+}} e^{-2 t p} w(t)^{-1} u(p) d t d p<\infty .
$$

Then, L is compact from $H_{K}(w)$ into $L_{2}(u)$ with norm less than or equal to $\sqrt{M}$.

Corollary 1. For $u, w \in L_{1}\left(\mathbb{R}^{+}\right)$, then, $L$ is compact from $H_{K}\left(w^{-1}\right)$ into $L_{2}(u)$. 
In the paper [41], there were given many concrete examples satisfying (28) and the conditions in Corollary 1 , and it was also derived real inversion formulas of the Laplace transform but in terms of the singular values decomposition and the Tikhonov regularization. This allowed a general numerical and real inversion formula of the Laplace transform.

Recall that the reproducing kernel Hilbert space $H_{K}(w)$ admits the concrete representation of the reproducing kernel

$$
K_{w}(s, t)=\int_{0}^{\min (s, t)} w(\xi)^{-1} d \xi .
$$

In order that $(29)$ is finite, we need to assume that

$$
\int_{0}^{1} \frac{d \xi}{w(\xi)}<\infty
$$

Under the situation, we obtained:

Theorem 5 ([13]). For positive continuous functions $u \in L_{1}(\mathbb{R})$ and $w$ on $[0, \infty)$ satisfying the conditions (28) and (30), the Moore-Penrose generalized inverse $g^{\dagger}$ of the (modified) Laplace transform $L$ from $H_{K}(w)$ into $L_{2}(u)$ exists uniquely if and only if it is the image of the derivative of a function in the space $H_{K}(w)$. Then, when we take a finite number of points $\left\{p_{j}\right\}_{j=1}^{n}$, an approximate inverse depending on the points $\left\{p_{j}\right\}_{j=1}^{n}$ is represented as follows:

$$
g_{A}^{\dagger}(x)=\sum_{j, j^{\prime}=1}^{n}\left(L^{*} g\right)\left(p_{j}\right) \widetilde{a_{j j^{\prime}}} L^{*} L K_{w}\left(p_{j^{\prime}}, x\right)
$$

where

$$
\begin{aligned}
\left(L^{*} g\right)\left(p_{j}\right) & =\int_{0}^{p_{j}} \mathscr{L}(g u)(t) \frac{d t}{w(t)}, \\
\left(L^{*} L K_{w}\left(p_{j}, x\right)\right) & =\int_{0}^{x} \int_{0}^{p_{j}} \frac{d t}{w(t)} \frac{d \tau}{w(\tau)}(\mathscr{L} u)(t+\tau),
\end{aligned}
$$

and $\widetilde{a_{j j^{\prime}}}$ are the elements of the inverse of the positive definite symmetric matrix constituted by the elements

$$
a_{j j^{\prime}}=\int_{0}^{p_{j}} \int_{0}^{p_{j^{\prime}}} \int_{0}^{\infty} \frac{(\mathscr{L} u)(t+\xi)(\mathscr{L} u)(\tau+\xi)}{w(\xi)} \frac{d t}{w(t)} \frac{d \tau}{w(\tau)} d \xi
$$

A pointwise estimation of the inverse is given in terms of the points $\left\{p_{j}\right\}_{j=1}^{n}$ by

$$
\left|g_{A}^{\dagger}(x)\right| \leq\left(\sum_{j, j^{\prime}=1}^{n}\left(L^{*} g\right)\left(p_{j}\right) \widetilde{a_{j j^{\prime}}}\left(L^{*} g\right)\left(p_{j^{\prime}}\right)\right)^{1 / 2}
$$




$$
\cdot\left(\sum_{j, j^{\prime}=1}^{n} L^{*} L K_{w}\left(p_{j}, x\right) \widetilde{a_{j j^{\prime}}} L^{*} L K_{w}\left(p_{j^{\prime}}, x\right)\right)^{1 / 2} .
$$

As a more powerful and practical method, we introduced the idea of the Tikhonov regularization.

\section{The Tikhonov Regularization}

We shall consider some practical and more concrete representation in the extremal functions involved in the Tikhonov regularization by using the theory of reproducing kernels.

Furthermore, we shall need error estimates when $\mathbf{d}$ contains error or noises. For this fundamental problem, we obtain the following conclusion.

Theorem 6. Let $L: H_{K} \rightarrow \mathscr{H}$ be a bounded linear operator, and define the inner product

$$
\left\langle f_{1}, f_{2}\right\rangle_{H_{K_{\lambda}}}=\alpha\left\langle f_{1}, f_{2}\right\rangle_{H_{K}}+\left\langle L f_{1}, L f_{2}\right\rangle_{\mathscr{H}}
$$

for $f_{1}, f_{2} \in H_{K}$. Then $\left(H_{K},\langle\cdot, \cdot\rangle_{H_{K \alpha}}\right)$ is a reproducing kernel Hilbert space whose reproducing kernel is given by

$$
K_{\alpha}(p, q)=\left[\left(\alpha+L^{*} L\right)^{-1} K_{q}\right](p) .
$$

Here, $K_{\alpha}(p, q)$ is the solution $\widetilde{K}_{\alpha}(p, q)$ of the functional equation

$$
\widetilde{K}_{\alpha}(p, q)+\frac{1}{\alpha}\left(L \widetilde{K}_{q}, L K_{p}\right)_{\mathscr{C}}=\frac{1}{\alpha} K(p, q),
$$

that is corresponding to the Fredholm integral equation of the second kind for many concrete cases. Moreover, we are using

$$
\widetilde{K}_{q}=\widetilde{K}_{\alpha}(\cdot, q) \in H_{K} \quad \text { for } \quad q \in E, \quad K_{p}=K(\cdot, p) \quad \text { for } \quad p \in E .
$$

Theorem 7. The map

$$
f \in H_{K} \mapsto\left\{\alpha\left\|f: H_{K}\right\|^{2}+\|L f-\mathbf{d}: \mathscr{H}\|^{2}\right\} \in \mathbb{R}
$$

attains the minimum and the minimum is attained only at $f_{\mathbf{d}, \alpha} \in H_{K}$ such that

$$
\left(f_{\mathbf{d}, \alpha}\right)(p)=\left\langle\mathbf{d}, L K_{\alpha}(\cdot, p)\right\rangle_{\mathscr{H}} .
$$

Furthermore, $\left(f_{\mathbf{d}, \alpha}\right)(p)$ satisfies

$$
\left|\left(f_{\mathbf{d}, \alpha}\right)(p)\right| \leq \sqrt{\frac{K(p, p)}{2 \alpha}}\|\mathbf{d}\|_{\mathscr{H}} .
$$


This theorem means that in order to obtain good approximate solutions, we must take a sufficiently small $\alpha$. However, here we have restrictions for them, as we see, when $\mathbf{d}$ moves to $\mathbf{d}^{\prime}$, by considering $f_{\mathbf{d}, \alpha}(p)-f_{\mathbf{d}^{\prime}, \alpha}(p)$ in connection with the relation of the difference $\left\|\mathbf{d}-\mathbf{d}^{\prime}\right\|_{\mathscr{H}}$. This fact is a very natural one, because we cannot obtain good solutions from the data containing errors. Here we wish to know how to take a small $\alpha$ a prior and what is the bound for it.

The basic idea may be given as follows. We examine for various $\alpha$ tending to zero, the corresponding extremal functions. By examining the sequence of the extremal functions, when it converges to some function numerically and after that when the sequence diverges numerically, it will give the bound for $\alpha$ numerically (see $[18,19])$.

For up-to-date versions of the Tikhonov regularization using the theory of reproducing kernels, see [7, 9].

\section{Real and Numerical Inversion Formula of the Laplace Transform by the Tikhonov Regularization}

We shall consider the inversion formula of the Laplace transform

$$
(\mathscr{L} F)(p)=f(p)=\int_{0}^{\infty} e^{-p t} F(t) d t, \quad p>0
$$

for some natural function spaces. For more general functions, we shall apply their transforms suitably in order to apply the results. In order to consider a bounded linear operator by the Laplace transform, we shall recall a natural function space from [37].

On the positive real line $\mathbb{R}^{+}$, we shall consider the norm

$$
\left\{\int_{0}^{\infty}\left|F^{\prime}(t)\right|^{2} \frac{1}{t} e^{t} d t\right\}^{1 / 2}
$$

for absolutely continuous functions $F$ satisfying $F(0)=0$. This space $H_{K}$ admits the reproducing kernel

$$
K\left(t, t^{\prime}\right)=\int_{0}^{\min \left(t, t^{\prime}\right)} \xi e^{-\xi} d \xi
$$

Then, we have

$$
\int_{0}^{\infty}|(\mathscr{L} F)(p) p|^{2} d p \leq \frac{1}{2}\|F\|_{H_{K}}^{2}
$$

That is, $(\mathscr{L} F)(p) p$ is a bounded linear operator from $H_{K}$ into $L_{2}\left(\mathbb{R}^{+}, d p\right)=$ $L_{2}\left(\mathbb{R}^{+}\right)$. By using this reproducing kernel Hilbert space, we obtain the next significative result, following our general method. 
Proposition 6. For any $g \in L_{2}\left(\mathbb{R}^{+}\right)$and for any $\alpha>0$, in the sense

$$
\begin{aligned}
\inf _{F \in H_{K}}\left\{\alpha \int_{0}^{\infty}\left|F^{\prime}(t)\right|^{2} \frac{1}{t} e^{t} d t+\|(\mathscr{L} F)(p) p-g\|_{L_{2}\left(\mathbb{R}^{+}\right)}^{2}\right\} \\
=\alpha \int_{0}^{\infty}\left|F_{\alpha, g}^{* \prime}(t)\right|^{2} \frac{1}{t} e^{t} d t+\left\|\left(\mathscr{L} F_{\alpha, g}^{*}\right)(p) p-g\right\|_{L_{2}\left(\mathbb{R}^{+}\right)}^{2}
\end{aligned}
$$

there exists a uniquely determined best approximate function $F_{\alpha, g}^{*}$ and it is represented by

$$
F_{\alpha, g}^{*}(t)=\int_{0}^{\infty} g(\xi)\left(\mathscr{L} K_{\alpha}(\cdot, t)\right)(\xi) \xi d \xi .
$$

Here, $K_{\alpha}(\cdot, t)$ is determined by the functional equation for $K_{\alpha, t^{\prime}}=K_{\alpha}\left(\cdot, t^{\prime}\right), K_{t}=$ $K(\cdot, t)$,

$$
K_{\alpha}\left(t, t^{\prime}\right)=\frac{1}{\alpha} K\left(t, t^{\prime}\right)-\frac{1}{\alpha}\left(\left(\mathscr{L} K_{\alpha, t^{\prime}}\right)(p) p,\left(\mathscr{L} K_{t}\right)(p) p\right)_{L_{2}\left(\mathbb{R}^{+}\right)}
$$

We calculate the approximate inverse $F_{\alpha, g}^{*}(t)$ by using (37). By taking the Laplace transform of (38) with respect to $t$, by changing the variables $t$ and $t^{\prime}$

$\left.\left(\mathscr{L} K_{\alpha}(\cdot, t)\right)(\xi)=\frac{1}{\alpha}(\mathscr{L} K(\cdot, t))(\xi)-\frac{1}{\alpha}\left(\left(\mathscr{L} K_{\alpha, t}\right)(p) p,(\mathscr{L}(\mathscr{L} K).(p) p)\right)(\xi)\right)_{L_{2}\left(\mathbb{R}^{+}\right)}$.

Here,

$$
\begin{gathered}
K\left(t, t^{\prime}\right)=\left\{\begin{array}{l}
-t e^{-t}-e^{-t}+1 \quad \text { for } t \leq t^{\prime} \\
-t^{\prime} e^{-t^{\prime}}-e^{-t^{\prime}}+1 \quad \text { for } t \geq t^{\prime}
\end{array}\right. \\
\left(\mathscr{L} K\left(\cdot, t^{\prime}\right)\right)(p)=e^{-t^{\prime} p} e^{-t^{\prime}}\left[\frac{-t^{\prime}}{p(p+1)}+\frac{-1}{p(p+1)^{2}}\right]+\frac{1}{p(p+1)^{2}}, \\
\int_{0}^{\infty} e^{-q t^{\prime}}\left(\mathscr{L} K\left(\cdot, t^{\prime}\right)\right)(p) d t^{\prime}=\frac{1}{p q(p+q+1)^{2}}
\end{gathered}
$$

Therefore, by setting as $\left(\mathscr{L} K_{\alpha}(\cdot, t)\right)(\xi) \xi=H_{\alpha}(\xi, t)$, we obtain the Fredholm integral equation of the second kind:

$$
\alpha H_{\alpha}(\xi, t)+\int_{0}^{\infty} \frac{H_{\alpha}(p, t)}{(p+\xi+1)^{2}} d p=-\frac{e^{-t \xi} e^{-t}}{\xi+1}\left(t+\frac{1}{\xi+1}\right)+\frac{1}{(\xi+1)^{2}},
$$

which is corresponding to (32). By solving this integral equation, H. Fujiwara derived a very reasonable numerical inversion formula for the integral transform and he expanded very good algorithms for numerical and real inversion formulas of the Laplace transform. Consequently, international patents by its software are being forwarded to Japan, USA, Canada and EU (for more detailed references and comments for this equation, see [40]).

Fig. 1 is an example for $\mathscr{L} F(p)=\exp (-p)$ for which $F(t)=\delta_{1}(t)$ in the distribution sense, and Fig. 2 is for 


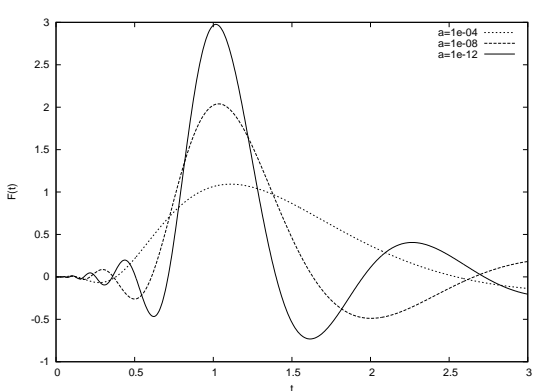

(a) $\alpha \geq 10^{-12}$

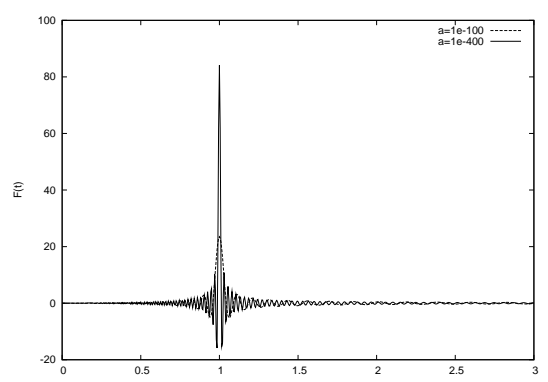

(b) $\alpha=10^{-100}, 10^{-400}$

Fig. 1 Numerical results for the delta function $\delta_{1}$

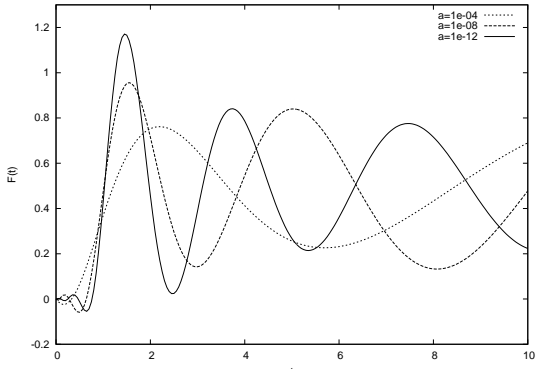

(a) $\alpha \geq 10^{-12}$

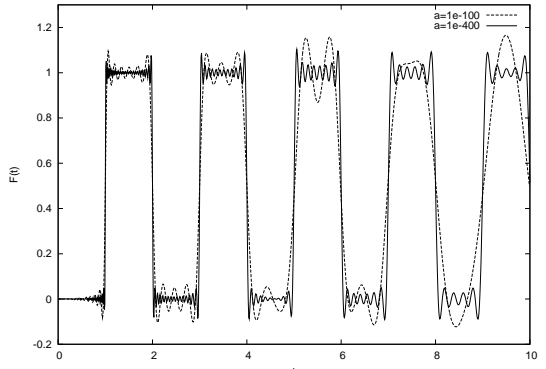

(b) $\alpha=10^{-100}, 10^{-400}$

Fig. 2 Numerical results for a square wave function

$$
\mathscr{L} F(p)=\frac{e^{-p}}{p\left(1+e^{-p}\right)}
$$

in which $F(t)$ is a square wave function.

In both figures, (a) is computed with large regularization parameters $\alpha \geq 10^{-12}$, and (b) is computed with small regularization parameters $\alpha=10^{-100}, 10^{-400}$. At this moment, theoretically we shall use the whole data of the output - in fact, 6000 data. Surprisingly enough, Fujiwara gave the solutions with $\alpha=10^{-400}$ and $\mathbf{6 0 0}$ digits precision. The core of the above mentioned and corresponding patent is $\mathbf{1 0}$ GB data for the solutions. See [17, 19, 38, 40, 41] for the basic references.

This great numerical power of Fujiwara has been giving a great impact within their associated numerical problems.

In [6], we gave an exact real inversion formula such that for any given finite number of points $\left\{p_{j}\right\}$ and for any given values $\left\{d_{j}\right\}$, we construct an optimal inversion $F$ satisfying $f\left(p_{j}\right)=d_{j}$ whose results are derived from the general concept of the next section. Moreover, it is interesting to realize that this concept was derived from a generalization of the Pythagorian theorem. 
Proposition 7. For any given $n$ values $\mathbf{d}=\left\{d_{j}\right\}_{j=1}^{n}$, among the Laplace transforms taking the values

$$
f\left(p_{j}\right)=d_{j}, \quad j=1,2, \ldots, n,
$$

and among their inverses, the uniquely determined function $F_{\mathbf{d}}^{*}(t)$ with the minimum norm $\|F\|_{H_{K}}$ is uniquely determined and it is represented by:

$$
F_{\mathbf{d}}^{*}(t)=\sum_{j, j^{\prime}=1}^{n}\left(d_{j} p_{j}\right) \widetilde{a}_{j j^{\prime}}\left(\mathbf{L}^{*} \mathbf{e}_{j^{\prime}}\right)(t) .
$$

Here, $\widetilde{a}_{j j^{\prime}}$ is the element of the positive definite Hermitian matrix formed by the element

$$
a_{j j^{\prime}}=\frac{1}{\left(p_{j}+p_{j^{\prime}}+1\right)^{2}}
$$

and

$$
\begin{aligned}
\left(\mathbf{L}^{*} \mathbf{e}_{j}\right)(t) & =\left(\left(\mathbf{L}^{*} \mathbf{e}_{j}\right)(\cdot), K(\cdot, t)\right)_{H_{K}} \\
& =\left(\mathbf{e}_{j}, \mathbf{L} K(\cdot, t)\right)_{\mathbb{R}^{n}} \\
& =e^{-t p_{j}} e^{-t}\left[\frac{-t}{\left(p_{j}+1\right)}+\frac{-1}{\left(p_{j}+1\right)^{2}}\right]+\frac{1}{\left(p_{j}+1\right)^{2}} .
\end{aligned}
$$

\section{Inversion Mapping from Many Kinds of Information Data}

We shall give a fundamental concept to inversions from many kinds of information data. For an abstract set $\Lambda$, we shall consider an operator-valued function $L_{\lambda}$ on $\Lambda$,

$$
\Lambda \longrightarrow L_{\lambda}
$$

where $L_{\lambda}$ are bounded linear operators from a Hilbert space $\mathscr{H}$ into various Hilbert spaces $\mathscr{H}_{\lambda}$,

$$
L_{\lambda}: \mathscr{H} \mapsto \mathscr{H}_{\lambda}
$$

In particular, we are interested in the inversion formula

$$
L_{\lambda} x \mapsto x, \quad x \in \mathscr{H}
$$

Here, we consider $\left\{L_{\lambda} x ; \lambda \in \Lambda\right\}$ as informations obtained from $x$ and we wish to determine $x$ from the informations. However, the informations $L_{\lambda} x$ belong to various Hilbert spaces $\mathscr{H}_{\lambda}$, and so, in order to unify the informations in a sense, we shall take fixed elements $\mathbf{b}_{\lambda, \omega} \in \mathscr{H}_{\lambda}$ and consider the linear mapping from $\mathscr{H}$,

$$
X_{\mathbf{b}}(\lambda, \omega)=\left(L_{\lambda} x, \mathbf{b}_{\lambda, \omega}\right)_{\mathscr{H}_{\lambda}}=\left(x, L_{\lambda}^{*} \mathbf{b}_{\lambda, \omega}\right) \mathscr{H}, \quad x \in \mathscr{H},
$$


into a linear space comprising functions on $\Lambda \times \Omega$. For the informations $L_{\lambda} x$, we shall consider $X_{\mathbf{b}}(\lambda, \omega)$ as observations (measurements, in fact) for $x$ depending on $\lambda$ and $\omega$. For this linear mapping (44), we form the positive definite quadratic function $K_{\mathbf{b}}\left(\lambda, \omega ; \lambda^{\prime}, \omega^{\prime}\right)$ on $\Lambda \times \Omega$ defined by

$$
K_{\mathbf{b}}\left(\lambda, \omega ; \lambda^{\prime}, \omega^{\prime}\right)=\left(L_{\lambda^{\prime}}^{*} \mathbf{b}_{\lambda^{\prime}, \omega^{\prime}}, L_{\lambda}^{*} \mathbf{b}_{\lambda, \omega}\right)_{\mathscr{H}}=\left(L_{\lambda} L_{\lambda^{\prime}}^{*} \mathbf{b}_{\lambda^{\prime}, \omega^{\prime}}, \mathbf{b}_{\lambda, \omega}\right)_{\mathscr{C} \lambda} \quad \text { on } \quad \Lambda \times \Omega .
$$

Then, we can apply our theory. The concept was derived from the generalization of the Pythagorean theorem in the following way (cf. [2, 33, 39]): Let $x \in \mathbb{R}^{n}$ and $\left\{\mathbf{e}_{j}\right\}_{j=1}^{n}$ be linearly independent unit vectors. We consider the linear mappings

$$
L: x \mapsto\left\{x-\left(x, \mathbf{e}_{j}\right) \mathbf{e}_{j}\right\}_{j=1}^{n}
$$

from $\mathbb{R}^{n}$ into $\mathbb{R}^{n}$. Then, we wish to establish an isometric identity and inversion formula for the operators. Recall the Pythagorean theorem for $n=2$. By our operator versions, we can establish the desired results.

Note that in (46), for $n \geq 3$, if we consider

$$
\left\{\left\|x-\left(x, \mathbf{e}_{j}\right) \mathbf{e}_{j}\right\|\right\}_{j=1}^{n}
$$

as scalar valued mappings, then the mappings are, in general, no longer linear. So, we must consider the operator valued mappings (46) in order to obtain isometric mappings in the framework of Hilbert spaces.

We see that some related equations were considered as follows [28, p. 128-157]: Let $H, H_{j}, j=1,2, \ldots, p$, be Hilbert spaces and let

$$
R_{j}: H \mapsto H_{j}, \quad j=1,2, \ldots, p,
$$

be linear continuous maps from $H$ onto $H_{j}$. Let $g_{j} \in H_{j}$ be given. Then, consider the problem to compute $f \in H$ such that

$$
R_{j} f=g_{j}, \quad j=1,2, \ldots, p .
$$

This equations are very important in the theory of computerized tomography by discretization. The typical method is Kaczmarz's method based on an iterative method by using the orthogonal projections $P_{j}$ in $H$ onto the affine subspaces $R_{j} f=g_{j}$. See [28] for the details.

As for our direct solutions for (49) it seems that the result is stable for the sake of (49) as data. 


\section{New Real Inversion Formulas of the Laplace Transform}

We shall state the new formulas by our new discretization method. The following theorem was derived directly from our general Theorem 1:

Theorem 8. For any fixed positive continuous function $w$ on $[0, \infty)$ satisfying the condition

$$
\int_{0}^{\infty} e^{-x t} \frac{d t}{w(t)}<\infty, \quad x>0,
$$

the best approximate inverse $F_{A}^{*}(t)$ of the Laplace transform

$$
(L F)(p)=\int_{0}^{\infty} e^{-t p} F(t) d t
$$

for any function $F \in L_{2}(w)$ satisfying

$$
\int_{0}^{\infty}|F(t)|^{2} w(t) d t<\infty
$$

is given by the formula by taking a finite number of points $\left\{p_{j}\right\}_{j=1}^{n}$

$$
F_{A}^{*}(t)=\sum_{j, j^{\prime}=1}^{n}(L F)\left(p_{j}\right) \widetilde{a_{j j^{\prime}}} \frac{e^{-p_{j^{\prime}} t}}{w(t)}
$$

where $\widetilde{a_{j j^{\prime}}}$ are the elements of the inverse of the positive definite symmetric matrix A constituted by the elements

$$
a_{j j^{\prime}}=\int_{0}^{\infty} e^{-\left(p_{j}+p_{j^{\prime}}\right) \xi} \frac{d \xi}{w(\xi)} .
$$

This function $F_{A}^{*}(t)$ is the optimal inverse in the sense that among the functions of the space $L_{2}(w)$ whose Laplace transforms are $(L F)\left(p_{j}\right)$ it minimizes the norms in $L_{2}(w)$.

In our approach of the numerical inversion formula of the Laplace transform, the effectiveness of the inversion may be reduced to some good selection of the weights $w$.

We shall list up several examples in this section from [11, 21]:

(1) For $w(t)=t^{1-\mu}$,

$$
L\left(\frac{1}{w}\right)(p)=\frac{1}{p^{\mu}} \Gamma(\mu), \quad \mu>0 .
$$

(2) For $w(t)=\frac{e^{t}}{t}$,

$$
L\left(\frac{1}{w}\right)(p)=\frac{1}{(p+1)^{2}} .
$$


(3) For $w(t)=(t+1)^{-2 n}$,

$$
L\left(\frac{1}{w}\right)(p)=\frac{(2 n) !}{(p+1)^{2 n+1}} e_{2 n}(p)
$$

for

$$
e_{k}(p)=1+p+\frac{p^{2}}{2 !}+\ldots+\frac{p^{k}}{k !} ; \quad n=1,2, \ldots .
$$

(4) For $w(t)=\frac{1+e^{t}}{t^{n-1}}$,

$$
L\left(\frac{1}{w}\right)(p)=(n-1) ! \sum_{k=1}^{\infty} \frac{(-1)^{k-1}}{(p+k)^{n}}, \quad p>-1, n=1,2, \ldots
$$

(5) For $w(t)=\frac{t}{1-\frac{t+2}{2 t}\left(1-e^{-t}\right)}$,

$$
L\left(\frac{1}{w}\right)(p)=-1+\left(p+\frac{1}{2}\right) \log \frac{p+1}{p} .
$$

(6) For $w(t)=\frac{t^{2}}{\left(e^{-t}-1\right)^{n}}$,

$L\left(\frac{1}{w}\right)(p)=\sum_{k=0}^{n}\left(\begin{array}{l}n \\ k\end{array}\right)(p+n-k) \log (p+n-k)(2 q+p) \log (2 q+p), \quad n=2,4$.

(7) For $w(t)=\frac{t^{3}}{\left(1-e^{-q t}\right)^{n}}$,

$L\left(\frac{1}{w}\right)(p)=\frac{1}{2} \sum_{k=2}^{n}(-1)^{k-1}\left(\begin{array}{l}n \\ k\end{array}\right)(p+k q)^{2} \log (p+k q), n>2, p>0, q n+p>0$.

(8) For $w(t)=\frac{t^{2}}{\left(1-e^{-q t}\right)^{2}}$,

$L\left(\frac{1}{w}\right)(p)=(2 q+p) \log (2 q+p)-2(p+q) \log (p+q)+p \log p, \quad p>0,2 q>-p$.

We shall now state very concrete examples from [44].

Example 1. As a first example take $p_{k}=k-\frac{1}{2}$. Then $a_{k l}=\frac{1}{k+l-1}$, and the matrix $A_{n}$ is the Hilbert matrix

$$
A_{n}=\left(\frac{1}{k+l-1}\right)_{n \times n} .
$$

The inverse of $A_{n}$ is well-known (see [5, 15]). In fact,

$$
\widetilde{a}_{l k}=(-1)^{k+l}(k+l-1)\left(\begin{array}{c}
n+k-1 \\
n-l
\end{array}\right)\left(\begin{array}{c}
n+l-1 \\
n-k
\end{array}\right)\left(\begin{array}{c}
k+l-2 \\
k-1
\end{array}\right)^{2} .
$$

Consequently, 


$$
\begin{aligned}
F_{A_{n}}^{*}(z)= & \sum_{k, l=1}^{n}(-1)^{k+l}(k+l-1) \\
& \cdot\left(\begin{array}{c}
n+k-1 \\
n-l
\end{array}\right)\left(\begin{array}{c}
n+l-1 \\
n-k
\end{array}\right)\left(\begin{array}{c}
k+l-2 \\
k-1
\end{array}\right)^{2} \frac{F\left(k-\frac{1}{2}\right)}{z+l-\frac{1}{2}} .
\end{aligned}
$$

Moreover,

$$
\begin{aligned}
\lim _{n \rightarrow \infty} \sum_{k, l=1}^{n}(-1)^{k+l}(k+l-1) \\
\quad \cdot\left(\begin{array}{c}
n+k-1 \\
n-l
\end{array}\right)\left(\begin{array}{c}
n+l-1 \\
n-k
\end{array}\right)\left(\begin{array}{c}
k+l-2 \\
k-1
\end{array}\right)^{2} \frac{F\left(k-\frac{1}{2}\right)}{z+l-\frac{1}{2}}=F(z), \quad \Re(z)>0,
\end{aligned}
$$

where the convergence is both pointwise and in $\mathscr{H}^{2}\left(\mathbb{C}_{+}\right)$norm, and the real variable inverse Laplace transform has the form

$$
\begin{aligned}
f(t)= & \lim _{n \rightarrow \infty} \sum_{k, l=1}^{n}(-1)^{k+l}(k+l-1) \\
& \cdot\left(\begin{array}{c}
n+k-1 \\
n-l
\end{array}\right)\left(\begin{array}{c}
n+l-1 \\
n-k
\end{array}\right)\left(\begin{array}{c}
k+l-2 \\
k-1
\end{array}\right)^{2} F\left(k-\frac{1}{2}\right) e^{-\left(l-\frac{1}{2}\right) t} .
\end{aligned}
$$

Example 2. Take now $p_{k}=k+\alpha / 2$ with $\alpha>-2$. Then $a_{k l}=\frac{1}{k+l+\alpha}$, and the matrix $A_{n}$ is a Hankel matrix

$$
A_{n}=\left(\frac{1}{k+l+\alpha}\right)_{n \times n} .
$$

The inverse of $A_{n}$ is less known in this case (see [5]). We have

$$
\begin{aligned}
\widetilde{a}_{l k}= & (-1)^{k+l}(k+l+\alpha)\left(\begin{array}{c}
n+k+\alpha \\
n-l
\end{array}\right)\left(\begin{array}{c}
n+l+\alpha \\
n-k
\end{array}\right) \\
& \cdot\left(\begin{array}{c}
k+l+\alpha-1 \\
k-1
\end{array}\right)\left(\begin{array}{c}
k+l+\alpha-1 \\
l-1
\end{array}\right) .
\end{aligned}
$$

Consequently,

$$
\begin{aligned}
F_{A_{n}}^{*}(z)= & \sum_{k, l=1}^{n}(-1)^{k+l}(k+l+\alpha)\left(\begin{array}{c}
n+k+\alpha \\
n-l
\end{array}\right)\left(\begin{array}{c}
n+l+\alpha \\
n-k
\end{array}\right) \\
& \cdot\left(\begin{array}{c}
k+l+\alpha-1 \\
k-1
\end{array}\right)\left(\begin{array}{c}
k+l+\alpha-1 \\
l-1
\end{array}\right) \frac{F(k+\alpha / 2)}{z+l+\alpha / 2} .
\end{aligned}
$$

Moreover,

$$
\lim _{n \rightarrow \infty} \sum_{k, l=1}^{n}(-1)^{k+l}(k+l+\alpha)\left(\begin{array}{c}
n+k+\alpha \\
n-l
\end{array}\right)\left(\begin{array}{c}
n+l+\alpha \\
n-k
\end{array}\right)
$$




$$
\cdot\left(\begin{array}{c}
k+l+\alpha-1 \\
k-1
\end{array}\right)\left(\begin{array}{c}
k+l+\alpha-1 \\
l-1
\end{array}\right) \frac{F(k+\alpha / 2)}{z+l+\alpha / 2}=F(z), \quad \Re(z)>0,
$$

where the convergence is both pointwise and in $\mathscr{H}^{2}\left(\mathbb{C}_{+}\right)$norm, and the real variable inverse Laplace transform has the form

$$
\begin{aligned}
f(t)= & \lim _{n \rightarrow \infty} \sum_{k, l=1}^{n}(-1)^{k+l}(k+l+\alpha)\left(\begin{array}{c}
n+k+\alpha \\
n-l
\end{array}\right)\left(\begin{array}{c}
n+l+\alpha \\
n-k
\end{array}\right) \\
& \cdot\left(\begin{array}{c}
k+l+\alpha-1 \\
k-1
\end{array}\right)\left(\begin{array}{c}
k+l+\alpha-1 \\
l-1
\end{array}\right) F(k+\alpha / 2) e^{-(l+\alpha / 2) t} .
\end{aligned}
$$

Example 3. Choose now $p_{k}=\frac{\alpha}{2}+\frac{1}{k}$. Then,

$$
A_{n}=\left(\frac{1}{\alpha+\frac{1}{k}+\frac{1}{l}}\right)_{n \times n}=\left(\frac{k l}{\alpha k l+k+l}\right)_{n \times n},
$$

and we have

$$
\begin{aligned}
\tilde{a}_{l k}^{n}=(-1)^{k+l} \frac{\prod_{j=1}^{n}\left[\left(\alpha+\frac{1}{j}+\frac{1}{k}\right)\left(\alpha+\frac{1}{j}+\frac{1}{l}\right)\right]}{\left(\alpha+\frac{1}{k}+\frac{1}{l}\right) \prod_{j=1}^{k-1}\left(\frac{1}{k}-\frac{1}{j}\right) \prod_{j=k+1}^{n}\left(\frac{1}{j}-\frac{1}{k}\right) \prod_{j=1}^{l-1}\left(\frac{1}{l}-\frac{1}{j}\right) \prod_{j=l+1}^{n}\left(\frac{1}{j}-\frac{1}{l}\right)} \\
=(-1)^{k+l} \frac{\prod_{j=1}^{n}(\alpha j k+j+k)(\alpha j l+j+l)}{\frac{\alpha k l+k+l}{k l} \cdot \frac{(-1)^{k-1}}{k^{k-1}} \cdot \frac{(-1)^{n-k}(n-k) !}{k^{n-k} \frac{n !}{k !}} \cdot \frac{(-1)^{l-1}}{l^{l-1}} \cdot \frac{(-1)^{n-l}(n-l) !}{l^{n-l} \frac{n !}{l !}} .} .
\end{aligned}
$$

Finally, we obtain

$$
\tilde{a}_{l k}^{n}=(-1)^{k+l} \frac{\prod_{j=1}^{n}[(\alpha j k+j+k)(\alpha j l+j+l)]}{(\alpha k l+k+l) k !(n-k) ! l !(n-l) !},
$$

and

$$
F_{A_{n}}^{*}(z)=\sum_{k, l=1}^{n}(-1)^{k+l} \frac{\prod_{j=1}^{n}[(\alpha j k+j+k)(\alpha j l+j+l)]}{(\alpha k l+k+l) k !(n-k) ! l !(n-l) !} \frac{F\left(\frac{1}{k}+\frac{\alpha}{2}\right)}{z+\frac{1}{l}+\frac{\alpha}{2}} .
$$

Moreover, 
$\lim _{n \rightarrow \infty} \sum_{k, l=1}^{n}(-1)^{k+l} \frac{\prod_{j=1}^{n}[(\alpha j k+j+k)(\alpha j l+j+l)]}{(\alpha k l+k+l) k !(n-k) ! l !(n-l) !} \frac{F\left(\frac{1}{k}+\frac{\alpha}{2}\right)}{z+\frac{1}{l}+\frac{\alpha}{2}}=F(z), \quad \Re(z)>0$,

where the convergence is both pointwise and in the $\mathscr{H}^{2}\left(\mathbb{C}_{+}\right)$norm, and the real variable inverse Laplace transform has the form

$$
f(t)=\lim _{n \rightarrow \infty} \sum_{k, l=1}^{n}(-1)^{k+l} \frac{\prod_{j=1}^{n}[(\alpha j k+j+k)(\alpha j l+j+l)]}{(\alpha k l+k+l) k !(n-k) ! l !(n-l) !} F\left(\frac{1}{k}+\frac{\alpha}{2}\right) e^{-\left(\frac{1}{l}+\frac{\alpha}{2}\right) t} .
$$

Therefore, the last formula recovers the Laplace inverse from data on a finite interval $\left(\frac{\alpha}{2}, \frac{\alpha}{2}+1\right]$.

\section{Paley-Wiener Spaces and Sobolev Spaces}

The Aveiro discretization method in mathematics is essentially the application of Theorem 1 by using the backward transform method (stated in detail below) to analytical problems like general linear differential equations and integral equations.

Then, we shall use basically the Paley-Wiener and the Sobolev reproducing kernel Hilbert spaces. So, we need to introduce these spaces and we will do it in a simpler and natural way.

We refer to J. R. Higgins [22] and F. Stenger [42] for the details in connection with the sampling theory. The method which is using the Paley-Wiener spaces is called the sinc method. At first we shall fix notations and basic results in the sampling theory following the book by F. Stenger [42] and at the same time we shall show the basic relation of the sampling theory and the theory of reproducing kernels. Let consider the following domain

$$
D_{h}=\left(-\frac{\pi}{h}, \frac{\pi}{h}\right)^{n}, \quad h>0 .
$$

We shall consider the Fourier transform for functions $F$ in $L_{2}\left(\mathbb{R}^{n}, D_{h}\right)$ by

$$
f(z)=\frac{1}{(2 \pi)^{n}} \int_{\mathbb{R}^{n}} \chi_{D_{h}}(\xi) F(\xi) e^{-i z \cdot \xi} d \xi .
$$

Here $\xi=\left(\xi_{1}, \xi_{2}, \ldots, \xi_{n}\right), d \xi=d \xi_{1} d \xi_{2} \ldots d \xi_{n}, z \cdot \xi=z_{1} \xi_{1}+z_{2} \xi_{2} \ldots z_{n} \xi_{n}$ and $\chi_{D_{h}}(\xi)$ is the characteristic function of the domain $D_{h}$.

In order to identify the image space following the theory of reproducing kernels, we form the reproducing kernel

$$
K_{h}(z, \bar{u})=\frac{1}{(2 \pi)^{n}} \int_{\mathbb{R}^{n}} \chi_{D_{h}}(\xi) e^{-i z \cdot \xi} \overline{e^{-i u \cdot \xi}} d \xi
$$




$$
=\prod_{v}^{n} \frac{1}{\pi\left(z_{v}-\bar{u}_{v}\right)} \sin \frac{\pi}{h} \frac{1}{\pi\left(z_{v}-\bar{u}_{v}\right)}
$$

and it forms the Paley-Wiener space, i.e., the image space of (56) is called the PaleyWiener space $W\left(\frac{\pi}{h}\right)$ comprised of all analytic functions of exponential type satisfying for each $v$, with some constant $C_{v}, z_{v} \rightarrow \infty$

$$
\left|f\left(z_{1}, \ldots, z_{v}, z_{v+1}, \ldots, z_{n}\right)\right| \leq C_{v} \exp \left(-\frac{\pi\left|z_{v}\right|}{h}\right)
$$

and that

$$
\int_{\mathbb{R}^{n}}|f(x)|^{2} d x<\infty
$$

Then, for $j=\left(j_{1}, j_{2}, \ldots, j_{n}\right) \in \mathbb{Z}^{n}$, we have the identity

$$
\frac{1}{(2 \pi)^{n}} \int_{\mathbb{R}^{n}}|F(\xi)|^{2} d \xi=h^{n} \sum_{j}|f(j h)|^{2}=\int_{\mathbb{R}^{n}}|f(x)|^{2} d x .
$$

This means that

$$
f(x)=\left(f(\cdot), K_{h}(\cdot, x)\right)_{H_{K_{h}}}=h^{n} \sum_{j} f(j h) K_{h}(j h, x)=\int_{\mathbb{R}^{n}} f(\xi) K_{h}(\xi, x) d \xi
$$

and the whole data $f(x)$ is represented by the discrete data $\{f(j h)\}_{j}$ (sampling theorem). For a general theory for the sampling theory and error estimates for some finite points $\{h j\}$, see [35].

Next, we shall introduce the Sobolev Hilbert spaces which we will be using. For this purpose, let $m>\frac{n}{2}$ be an integer, and denote by ${ }_{N} C_{K}$ the binomial coefficient. Then, we have

$$
W^{m, 2}\left(\mathbb{R}^{n}\right)=H_{K}\left(\mathbb{R}^{n}\right),
$$

where $W^{m, 2}\left(\mathbb{R}^{n}\right)$ denotes the Sobolev space whose norm is given by

$$
\|F\|_{W^{m, 2}\left(\mathbb{R}^{n}\right)}=\sqrt{\sum_{v=0}^{m}{ }_{m} C_{v}\left(\sum_{\alpha \in \mathbb{Z}_{+}^{n},|\alpha| \leq v}^{v} \frac{v !}{\alpha !} \int_{\mathbb{R}^{n}}\left|\frac{\partial^{v} F(x)}{\partial x^{v}}\right|^{2} d x\right)}
$$

and

$$
K(x, y)=\frac{1}{(2 \pi)^{n}} \int_{\mathbb{R}^{n}} \frac{\exp (i(x-y) \cdot \xi)}{\left(1+|\xi|^{2}\right)^{m}} d \xi .
$$

In particular, note that if $m>\frac{n}{2}$ then $W^{m, 2}\left(\mathbb{R}^{n}\right)$ is embedded into $\mathrm{BC}\left(\mathbb{R}^{n}\right)$.

A generalization of the above spaces is given in the following way when considering $s>\frac{n}{2}$. Define 


$$
K(x, y):=\frac{1}{(2 \pi)^{n}} \int_{\mathbb{R}^{n}}\left(1+|\xi|^{2}\right)^{-s} \exp (i(x-y) \cdot \xi) d \xi .
$$

Then we have

$$
H_{K}\left(\mathbb{R}^{n}\right)=H^{s}\left(\mathbb{R}^{n}\right)
$$

where the norm is given by

$$
\|f\|_{H^{s}\left(\mathbb{R}^{n}\right)}=\left(\int_{\mathbb{R}^{n}}\left(1+|\xi|^{2}\right)^{s}|\mathscr{F} f(\xi)|^{2} d \xi\right)^{\frac{1}{2}} .
$$

We can now point out a very simple example. The space $H_{S}(\mathbb{R})$ is made up of absolutely continuous functions $F$ on $\mathbb{R}$ with the norm

$$
\|F\|_{H_{S}(\mathbb{R})}:=\sqrt{\int_{\mathbb{R}}\left(F(x)^{2}+F^{\prime}(x)^{2}\right) d x} .
$$

The Hilbert space $H_{S}(\mathbb{R})$ admits the reproducing kernel

$$
K(x, y):=\frac{1}{2 \pi} \int_{\mathbb{R}} \frac{1}{1+\xi^{2}} \exp (i(x-y) \xi) d \xi=\frac{1}{2} e^{-|x-y|} .
$$

Note, in particular, when constructing the reproducing kernel associated with the Paley-Wiener spaces and the Sobolev spaces the only significant difference appear in the change of the characteristic functions by the factors or weight $\left(1+|\xi|^{2}\right)^{-s}$.

In the sequel, we shall introduce typical results following our new discretization method.

\section{Ordinary Linear Differential Equations}

Ordinary differential equations is certainly a subarea of Mathematics having a very significant number applications to the real world. There are plenty of examples of their use in Physics, but also in Chemistry, Biology, Economics, etc.. Unfortunately, despite the great amount of applications, only the simplest ordinary differential equations admit solutions given by explicit formulas. However, some properties of the solutions of a given differential equation may be determined without finding their exact form. If a self-contained formula for the solution is not available, the solution may be numerically approximated by using computers if a suitable numerical method is implemented.

The argument presented here will show that our results will be valid in a very general situation. However, to state the results and methods in a simpler manner, we consider the following prototype differential operator:

$$
L y:=\alpha y^{\prime \prime}+\beta y^{\prime}+\gamma y .
$$


Here, we shall consider a very general situation in which the coefficients are arbitrary functions on their nature and on a general interval $I$.

We wish to construct some natural solution of

$$
L y=g
$$

for a very general function $g$ on a general interval $I$.

For a practical construction of the solution, we obtain the following formula.

Theorem 9. Let us fix a positive number $h$ and take a finite number of points $\left\{t_{j}\right\}_{j=1}^{n}$ of I such that

$$
\left(\alpha\left(t_{j}\right), \beta\left(t_{j}\right), \gamma\left(t_{j}\right)\right) \neq 0
$$

for each $j$. Then, the optimal solution $y_{h}^{A}$ of the equation (66) is given by

$$
y_{h}^{A}(t)=\frac{1}{2 \pi} \int_{-\pi / h}^{\pi / h} F_{h}^{A}(\xi) e^{-i t \xi} d \xi
$$

in terms of the function $F_{h}^{A} \in L_{2}(-\pi / h,+\pi / h)$ in the sense that $F_{h}^{A}$ has the minimum norm in $L_{2}(-\pi / h,+\pi / h)$ among the functions $F \in L_{2}(-\pi / h,+\pi / h)$ satisfying, for the characteristic function $\chi_{h}(t)$ of the interval $(-\pi / h,+\pi / h)$ :

$$
\frac{1}{2 \pi} \int_{\mathbb{R}} F(\xi)\left[\alpha(t)\left(-\xi^{2}\right)+\beta(t)(-i \xi)+\gamma(t)\right] \chi_{h}(\xi) \exp (-i t \xi) d \xi=g(t)
$$

for all $t=t_{j}$ and for the function space $L_{2}(-\pi / h,+\pi / h)$.

The best extremal function $F_{h}^{A}$ is given by

$$
F_{h}^{A}(\xi)=\sum_{j, j^{\prime}=1}^{n} g\left(t_{j}\right) \widetilde{a_{j j^{\prime}}} \overline{\left(\alpha\left(t_{j^{\prime}}\right)\left(-\xi^{2}\right)+\beta\left(t_{j^{\prime}}\right)(-i \xi)+\gamma\left(t_{j^{\prime}}\right)\right)} \exp \left(i t_{j^{\prime}} \xi\right) .
$$

Here, we are indirectly using the matrix $A=\left\{a_{j j^{\prime}}\right\}_{j, j^{\prime}=1}^{n}$ formed by the elements

$$
a_{j j^{\prime}}=K_{h h}\left(t_{j}, t_{j^{\prime}}\right)
$$

with

$$
\begin{aligned}
K_{h h}\left(t, t^{\prime}\right)= & \frac{1}{2 \pi} \int_{\mathbb{R}}\left[\alpha(t)\left(-\xi^{2}\right)+\beta(t)(-i \xi)+\gamma(t)\right] \overline{\left[\alpha\left(t^{\prime}\right)\left(-\xi^{2}\right)+\beta\left(t^{\prime}\right)(-i \xi)+\gamma\left(t^{\prime}\right)\right]} \\
& \cdot \chi_{h}(\xi) \exp \left(-i\left(t-t^{\prime}\right) \xi\right) d \xi
\end{aligned}
$$

which is positive definite and the elements $\widetilde{a_{j j^{\prime}}}$ directly used in (68) are the elements of the inverse of $\bar{A}$ (the complex conjugate of $A$ ).

Therefore, the optimal solution $y_{h}^{A}$ of the equation (66) is given by

$$
y_{h}^{A}(t)=\sum_{j, j^{\prime}=1}^{n} g\left(t_{j}\right) \widetilde{a_{j j^{\prime}}} \frac{1}{2 \pi}\left[-\overline{\alpha\left(t_{j^{\prime}}\right)} \int_{-\frac{\pi}{h}}^{\frac{\pi}{h}} \xi^{2} e^{-i\left(t-t_{j^{\prime}}\right) \xi} d \xi\right.
$$




$$
\left.+i \overline{\beta\left(t_{j^{\prime}}\right)} \int_{-\frac{\pi}{h}}^{\frac{\pi}{h}} \xi e^{-i\left(t-t_{j^{\prime}}\right) \xi} d \xi+\overline{\gamma\left(t_{j^{\prime}}\right)} \int_{-\frac{\pi}{h}}^{\frac{\pi}{h}} e^{-i\left(t-t_{j^{\prime}}\right) \xi} d \xi\right] .
$$

We shall refer to the situation of the theorem. At first, we are considering approximate solutions of the differential equation (66) and, at this point, we are considering the Paley-Wiener function spaces with parameter $h$ as the approximating function spaces. The function spaces are formed by analytic functions of the entire functions of exponential type that are decreasing to zero within exponential orders. Next, by using the Fourier inversion, the differential equation (66) may be transformed into (67). However, to solve the integral equation (67) is very difficult for the generality of the coefficient functions. So, we assume that (67) is valid on some finite number of points $t_{j}$. This assumption will be very reasonable for the discretization of the integral equation. By this assumption we can obtain an optimal approximate solution in a very simple way, by the fundamental Theorem 1 .

Here, we assume that equation (66) is valid on $I$ and so, as some practical case we would like to consider the equation in (66) on $I$ satisfying some boundary conditions. In the present case, the boundary conditions are given as zero at infinity for $I=\mathbb{R}$.

However, our result gives the approximate general solutions satisfying boundary values. For example, for a finite interval $(a, b)$, we consider $t_{1}=a$ and $t_{n}=b$ and $\alpha\left(t_{1}\right)=\beta\left(t_{1}\right)=\alpha\left(t_{n}\right)=\beta\left(t_{n}\right)=0$. Then, we can obtain the approximate solution having the arbitrary given boundary values $y_{h}^{A}\left(t_{1}\right)$ and $y_{h}^{A}\left(t_{n}\right)$. In addition, by a simple modification we may give the general approximate solutions satisfying the corresponding boundary values.

For a finite interval case $I$, following the boundary conditions, we can consider the corresponding reproducing kernels by the Sobolev Hilbert spaces. However, the concrete representations of the reproducing kernels are involved and depending on the boundary conditions. However, despite this great generality of the problem, we can still consider them and we can use them - as we shall see.

As a easily understandable circunstancie, for a smaller $h$ we can obtain better approximate solutions. in addition, for the representation (69) of the reproducing kernel $K_{h h}\left(t, t^{\prime}\right)$, we can calculate it easily.

As a very surprising fact, which in a sense exhibits the power of the method, we would like to point out that for variable coefficients linear differential equations, we can represent their approximate solutions satisfying their boundary conditions without using integrals.

\section{Linear Partial Differential Equations}

Partial differential equations (PDEs) arise frequently in the formulation of fundamental laws of nature and in the mathematical analysis of a wide variety of problems in applied mathematics, mathematical physics, and engineering science. This subject plays a central role in a modern mathematical sciences, especially in physics, 
geometry and analysis. Many problems of physicals interest are described by partial partial differential equations with appropriate initial and/or boundary conditions.

Typically, a given PDE will only be accessible by means of numerical solutions, and analytic solutions in a practical or research scenario are often impossible. However, it is vital to understand the general theory in order to conduct a sensible investigation. For example, we may need to understand what type of PDE we have to ensure the numerical solution is valid. Indeed, certain types of equations need appropriate boundary conditions; without a knowledge of the general theory it is possible that the problem may be ill-posed and the solution will be erroneous.

\subsection{Second Order Differential Equations}

The argument presented here will show that our results will valid in a very general situation. However, in order to state the results and methods in a simpler manner, we shall start by considering the following prototype of partial differential operator of (hyperbolic) type:

$$
L u:=\vartheta \frac{\partial^{2} u}{\partial t^{2}}-\alpha \frac{\partial^{2} u}{\partial x^{2}}-\gamma u
$$

Here, we shall consider a very general situation that the coefficients are arbitrary functions in time $t$ and space $x$ on a general domain $D_{T, I}=(0, T) \times I$, where $I$ is any interval. We wish, for example, to construct some natural solution of

$$
L u(t, x)=g(t, x), \quad(t, x) \in(0, T) \times I,
$$

satisfying the boundary condition

$$
u(t, x)=0, \quad(t, x) \in(0, T) \times \partial I,
$$

and the initial conditions

$$
u(0, x)=u_{0}(x), \quad x \in I
$$

and

$$
u_{t}(0, x)=v_{0}(x), x \in I,
$$

for very general functions $g, u_{0}$ and $v_{0}$ on a general domain $D_{T, I}$.

For a practical construction of the solution, we obtain the following formula.

Theorem 10. Let us fix a positive number $h$ and take a finite number of points $\left\{\left(t_{l}, x_{j}\right)\right\}_{l, j=1}^{n}$ of $D_{T, I}$ such that

$$
\left(\vartheta\left(t_{l}, x_{j}\right), \alpha\left(t_{l}, x_{j}\right), \gamma\left(t_{l}, x_{j}\right)\right) \neq 0
$$

as vectors, for each $(l, j)$. Then, the optimal solution $u_{h}^{A}$ of the equation (71) is given by $($ for $X=(t, x))$ : 


$$
u_{h}^{A}(t, x)=\frac{1}{(2 \pi)^{2}} \int_{D_{h}} F_{h}^{A}(\xi) e^{-i X \cdot \xi} d \xi
$$

in terms of the function $F_{h}^{A} \in L_{2}\left(D_{h}\right), D_{h}=((-\pi / h,+\pi / h) \times(-\pi / h,+\pi / h))$ in the sense that $F_{h}^{A}$ has the minimum norm in $L_{2}\left(D_{h}\right)$ among the functions $F \in L_{2}\left(D_{h}\right)$ satisfying, for the characteristic function $\chi_{D_{h}}(\xi)$ of $D_{h}$ :

$$
\begin{array}{r}
\frac{1}{(2 \pi)^{2}} \int_{\mathbb{R}^{2}} F(\xi)\left[\vartheta(t, x)\left(-\xi_{1}^{2}\right)-\alpha(t, x)\left(-\xi_{2}^{2}\right)-\gamma(t, x)\right] \chi_{D_{h}}(\xi) \exp (-i X \cdot \xi) d \xi \\
=g(t, x)(72)
\end{array}
$$

for all $X=X_{l j}$, i.e., $(t, x)=\left(t_{l}, x_{j}\right)$ and for the function space $L_{2}\left(D_{h}\right)$.

The best extremal function $F_{h}^{A}$ is given by

$$
\begin{aligned}
& F_{h}^{A}(\xi)=\sum_{l, j, l^{\prime}, j^{\prime}=1}^{n} g\left(t_{l}, x_{j}\right) \widetilde{a_{l j, l^{\prime} j^{\prime}}} \\
& \overline{\cdot\left(\vartheta\left(t_{l^{\prime}}, x_{j^{\prime}}\right)\left(-\xi_{1}^{2}\right)-\alpha\left(t_{t^{\prime}}, x_{j^{\prime}}\right)\left(-\xi_{2}^{2}\right)-\gamma\left(t_{l^{\prime}}, x_{j^{\prime}}\right)\right)} \exp \left(i X_{l^{\prime} j^{\prime}} \cdot \xi\right) .
\end{aligned}
$$

Here, we are using the matrix $A=\left\{a_{\imath j, l^{\prime} j^{\prime}}\right\}\left(l, l^{\prime}, j, j^{\prime}=1, \ldots, n\right)$ formed by the elements

$$
a_{\imath j, l^{\prime} j^{\prime}}=K_{h h}\left(X_{l j}, X_{l^{\prime} j^{\prime}}\right)
$$

with

$$
\begin{aligned}
& K_{h h}\left(X, X^{\prime}\right)=\frac{1}{(2 \pi)^{2}} \int_{\mathbb{R}^{2}}\left[\vartheta(t, x)\left(-\xi_{1}^{2}\right)-\alpha(t, x)\left(-\xi_{2}^{2}\right)-\gamma(t, x)\right] \\
& \left.. \overline{\left[\vartheta\left(t^{\prime}, x^{\prime}\right)\left(-\xi_{1}^{2}\right)-\alpha\left(t^{\prime}, x^{\prime}\right)\left(-\xi_{2}^{2}\right)-\gamma\left(t^{\prime}, x^{\prime}\right)\right]} \chi_{D_{h}}(\xi) \exp \left(-i\left(X-X^{\prime}\right) \cdot \xi\right)\right) d \xi
\end{aligned}
$$

which is positive definite and the $\widetilde{a_{i j, l^{\prime} j^{\prime}}}$ are the elements of the inverse of $\bar{A}$ (the complex conjugate of $A$ ).

Therefore, the optimal solution $u_{h}^{A}$ of the equation (71) is given by

$$
\begin{aligned}
u_{h}^{A}(t, x)= & \sum_{l, j, l^{\prime}, j^{\prime}=1}^{n} g\left(t_{l}, x_{j}\right) \widetilde{a_{l j, l^{\prime} j^{\prime}}} \\
& \frac{1}{(2 \pi)^{2}}\left[-\overline{\vartheta\left(t_{l^{\prime}}, x_{j^{\prime}}\right)} \int_{-\frac{\pi}{h}}^{\frac{\pi}{h}} \xi_{1}^{2} e^{-i\left(t-t_{l^{\prime}}\right) \xi_{1}} \int_{-\frac{\pi}{h}}^{\frac{\pi}{h}} e^{-i\left(x-x_{j^{\prime}}\right) \xi_{2}} d \xi_{2} d \xi_{1}\right. \\
& +\overline{\alpha\left(t_{l^{\prime}}, x_{j^{\prime}}\right)} \int_{-\frac{\pi}{h}}^{\frac{\pi}{h}} e^{-i\left(t-t_{l^{\prime}}\right) \xi_{1}} \int_{-\frac{\pi}{h}}^{\frac{\pi}{h}} \xi_{2}^{2} e^{-i\left(x-x_{j^{\prime}}\right) \xi_{2}} d \xi_{2} d \xi_{1} \\
& \left.-\overline{\gamma\left(t_{l^{\prime}}, x_{j^{\prime}}\right)} \int_{-\frac{\pi}{h}}^{\frac{\pi}{h}} e^{-i\left(t-t_{l^{\prime}}\right) \xi_{1}} \int_{-\frac{\pi}{h}}^{\frac{\pi}{h}} e^{-i\left(x-x_{j^{\prime}}\right) \xi_{2}} d \xi_{2} d \xi_{1}\right] .
\end{aligned}
$$

In the next result, we present the optimal solution for a particular typical case of equation (71). 
Corollary 2. If $\vartheta(t, x) \equiv 1, \alpha(t, x) \equiv 1, \gamma \equiv 0$ then equation (71) reduces to an inhomogeneous wave equation, and then the optimal solution of (71) is given as

$$
\begin{aligned}
u_{h}^{A}(t, x)= & \sum_{l, j, l^{\prime}, j^{\prime}=1}^{n} g\left(t_{l}, x_{j}\right) \widetilde{a_{l j, l^{\prime}} j^{\prime}} \frac{1}{(2 \pi)^{2}}\left[-\int_{-\frac{\pi}{h}}^{\frac{\pi}{h}} \xi_{1}^{2} e^{-i\left(t-t_{l^{\prime}}\right) \xi_{1}} \int_{-\frac{\pi}{h}}^{\frac{\pi}{h}} e^{-i\left(x-x_{j^{\prime}}\right) \xi_{2}} d \xi_{2} d \xi\right. \\
& \left.+\int_{-\frac{\pi}{h}}^{\frac{\pi}{h}} e^{-i\left(t-t_{l^{\prime}}\right) \xi_{1}} \int_{-\frac{\pi}{h}}^{\frac{\pi}{h}} \xi_{2}^{2} e^{-i\left(x-x_{j^{\prime}}\right) \xi_{2}} d \xi_{2} d \xi_{1}\right] .
\end{aligned}
$$

We shall refer to the situation of Theorem 10. At first, we are considering approximate solutions of the differential equation (71) and at this point, we are considering the Paley-Wiener function spaces with parameter $h$ as approximating function spaces. So, the differential operator (70) for these function spaces is well defined. Next, by using the Fourier inversion, the differential equation (71) may be transformed into (72). However, to solve the integral equation (72) is very difficult for the generality of the coefficient functions. Therefore, as previously exposed, we assume that (72) is valid on some finite number of points $\left(t_{l}, x_{j}\right)$. This assumption will be very reasonable for the discretization of the integral equation. By this assumption we can obtain an optimal approximate solutions in a very simple way.

Here, we assume that equation (71) is valid on $D_{T, I}$ and so, as some practical case we would like to consider the equation in (71) on $D_{T, I}$ satisfying some initial and boundary conditions. In the present case, the boundary conditions are given as zero at infinity for $I=\mathbb{R}$ by the property of the Paley-Wiener spaces.

Since the functions in the Paley-Wiener spaces are decreasing exponentially, for the zero boundary condition solutions, for a large interval $I$, Theorem 10 will give good approximate solutions. If $h$ is smaller, then we can consider the interval $I$ as a smaller one.

However, our result gives the approximate general solutions satisfying initial and boundary conditions. For example, for a finite interval $I=(a, b)$, we consider $t_{1}=0$ and

$$
\vartheta\left(0, x_{j}\right)=\alpha\left(0, x_{j}\right)=0 .
$$

Then, $\gamma\left(0, x_{j}\right) \neq 0$ and we have

$$
\gamma\left(0, x_{j}\right) u\left(0, x_{j}\right)=g\left(0, x_{j}\right) \Rightarrow u_{0}(x)=\frac{g\left(0, x_{j}\right)}{\gamma\left(0, x_{j}\right)} .
$$

Thus, we see that we can consider the initial condition to be given in a very general situation. additionally, we realize that we can obtain the approximate solution having the boundary and initial conditions from the above formulations.

However, in the above formula, we cannot give the initial velocity $v_{0}\left(x_{j}\right)$ at the same point $x_{j}$ but we will further refer to this problem. 


\subsubsection{When we give Initial Values and Initial Velocities}

In Theorem 10, we cannot give initial values and initial velocities at the same points $x_{j}$ and so, we shall now consider precisely this situation and will give a formula giving the initial values and initial velocities at the same points $x_{j}$.

We are considering the integral representation, in (72),

$$
\begin{aligned}
u(t, x)= & \frac{1}{(2 \pi)^{2}} \int_{\mathbb{R}^{2}} F(\xi)\left[\vartheta(t, x)\left(-\xi_{1}^{2}\right)-\alpha(t, x)\left(-\xi_{2}^{2}\right)-\gamma(t, x)\right] \\
& \chi_{D_{h}}(\xi) \exp (-i X \cdot \xi) d \xi .
\end{aligned}
$$

In order to consider the derivative $u_{t}(t, x)$, we shall assume the time independent coefficients case (however, by assuming the time differentiability for the coefficients, we can derive a similar, but a more complicated formula). Then, we have

$$
u(t, x)=\frac{1}{(2 \pi)^{2}} \int_{\mathbb{R}^{2}} F(\xi)\left[\vartheta(x)\left(-\xi_{1}^{2}\right)-\alpha(x)\left(-\xi_{2}^{2}\right)-\gamma(x)\right] \chi_{D_{h}}(\xi) \exp (-i X \cdot \xi) d \xi
$$

and

$$
\begin{aligned}
u_{t}(t, x)= & \frac{1}{(2 \pi)^{2}} \int_{\mathbb{R}^{2}} F(\xi)\left[\vartheta(x)\left(-\xi_{1}^{2}\right)-\alpha(x)\left(-\xi_{2}^{2}\right)-\gamma(x)\right] \\
& \left(-i \xi_{1}\right) \chi_{D_{h}}(\xi) \exp (-i X \cdot \xi) d \xi .
\end{aligned}
$$

Therefore, by considering the linear mappings (72) and (76) from $L_{2}\left(D_{h}\right)$ into

$$
\left\{u\left(t_{l}, x_{j}\right), l, j=1,2, \ldots, n \text {, and } u_{t}\left(0, x_{j}\right), j=1,2, \ldots, n\right\},
$$

we can obtain the desired formula, similarly.

Furthermore, note that we are giving the approximate solution for the homogeneous equation $(g \equiv 0)$ satisfying boundary and initial conditions.

The surprising fact in here is that for variable coefficients linear partial differential equations, we can represent their approximate solutions satisfying their initial and boundary conditions.

\subsubsection{Construction of Approximate Solutions and Proof of Theorem 10}

Since the structure of the Hilbert space $H_{K_{h h}}$ in Theorem 10 is -in general- very complicated and abstract, we shall approximate the space by taking a finite number of points and by using matrix theory. In order to realize approximately the inner product in the space $H_{K_{h h}}$ with some practical sense, we shall consider the natural and general approximate realization of the space $H_{K_{h h}}$.

By taking a finite number of points $\left\{\left(t_{l}, x_{j}\right)\right\}_{l, j=1}^{n}$, we set

$$
K_{h h}\left(X_{l j}, X_{l^{\prime} j^{\prime}}\right):=a_{l j, l^{\prime} j^{\prime}}
$$


and we will define $A:=\left[a_{l j, l^{\prime} j^{\prime}}\right]_{l, j=1}^{n}$. Then, if the matrix $A$ is positive definite, the corresponding norm in $H_{A}$ comprising the vectors

$$
\mathbf{y}=\left(y_{11}, y_{12}, \ldots, y_{1 n}, y_{21}, y_{22}, \ldots, y_{2 n}, \ldots, y_{n 1}, y_{n 2}, \ldots, y_{n n}\right)
$$

is determined by

$$
\|\mathbf{y}\|_{H_{A}}^{2}=\mathbf{y}^{*} \widetilde{A} \mathbf{y}
$$

where $\widetilde{A}:=\left[\widetilde{a_{l j, l^{\prime} j^{\prime}}}\right]$ (see $[35$, p. 250]).

Now, from (71) we set

$$
a_{\imath j, l^{\prime} j^{\prime}}=K_{h h}\left(X_{\imath j}, X_{l^{\prime} j^{\prime}}\right), \quad l, \iota^{\prime}, j, j^{\prime}=1, \ldots, n .
$$

If $A$ is positive definite, then the minimum norm solution $F_{h}^{A}(\xi)$ of the integral transform (72),

$$
F \in L_{2}\left(D_{h}\right) \longrightarrow H_{K_{h h}}
$$

is given by the following formula (having in mind our fundamental Theorem 1):

$$
\begin{aligned}
& F_{h}^{A}(\xi)=\sum_{l, j, l^{\prime}, j^{\prime}=1}^{n} g\left(t_{l}, x_{j}\right) \widetilde{a_{l j, l^{\prime} j^{\prime}}} \\
& \overline{\left(\vartheta\left(t_{t^{\prime}}, x_{j^{\prime}}\right)\left(-\xi_{1}^{2}\right)-\alpha\left(t_{l^{\prime}}, x_{j^{\prime}}\right)\left(-\xi_{2}^{2}\right)-\gamma\left(t_{t^{\prime}}, x_{j^{\prime}}\right)\right)} \exp \left(i X_{l^{\prime} j^{\prime}} \cdot \xi\right) .
\end{aligned}
$$

Associated with (72), it remains to observe that

$$
\left[\vartheta\left(t_{l}, x_{j}\right)\left(-\xi_{1}^{2}\right)-\alpha\left(t_{l}, x_{j}\right)\left(-\xi_{2}^{2}\right)-\gamma\left(t_{l}, x_{j}\right)\right] \exp \left(-i X_{l j} \cdot \xi\right), \quad l, j=1,2, \ldots, n,
$$

are linearly independent in the space $L_{2}\left(D_{h}\right)$ if and only if

$$
\left(\vartheta\left(t_{l}, x_{j}\right), \alpha\left(t_{l}, x_{j}\right), \gamma\left(t_{l}, x_{j}\right)\right) \neq 0
$$

as vectors, for each $\imath, j$.

We can directly prove this proposition by mathematical induction but, instead, in here, we shall prove it by a different method using associated differential operators, cf. [12]. In that paper, Y. Sawano gave the noble proof in the following reasoning. Assume that

$$
\sum_{l, j=1}^{n} P_{l, j}\left\{\vartheta\left(t_{l}, x_{j}\right)\left(-\xi_{1}^{2}\right)-\alpha\left(t_{l}, x_{j}\right)\left(-\xi_{2}^{2}\right)-\gamma\left(t_{l}, x_{j}\right)\right\} \exp \left(-i X_{l j} \cdot \xi\right) \equiv 0
$$

For each $\imath, j$, consider the differential operator

$$
P_{l, j}(D)=\left(\frac{d}{d \xi_{1}}+i t_{l}\right)^{3}\left(\frac{d}{d \xi_{2}}+i x_{j}\right)^{3}
$$

Then, since a well-known theorem about ordinary differential equations shows that 


$$
\operatorname{Ker}\left(\Pi_{l, j}^{n} P_{l, j}(D)\right)=\oplus_{l, j=1}^{n} \operatorname{Ker}\left(P_{l, j}(D)\right)
$$

in the sense of direct sum, we have

$$
P_{l, j}\left\{\vartheta\left(t_{l}, x_{j}\right)\left(-\xi_{1}^{2}\right)-\alpha\left(t_{l}, x_{j}\right)\left(-\xi_{2}^{2}\right)-\gamma\left(t_{l}, x_{j}\right)\right\} \exp \left(-i X_{l j} \cdot \xi\right) \equiv 0
$$

for each $\imath, j$ and hence

$$
P_{l, j}\left\{\vartheta\left(t_{l}, x_{j}\right)\left(-\xi_{1}^{2}\right)-\alpha\left(t_{l}, x_{j}\right)\left(-\xi_{2}^{2}\right)-\gamma\left(t_{l}, x_{j}\right)\right\} \equiv 0,
$$

This shows the desired result that $P_{l, j}=0$ for $l, j=1,2, \ldots, n$.

Hence, we obtain the main Theorem 10.

The argument presented in here will show that our results will be valid in a very general situation. However, in order to state the results and methods in a simpler manner, we shall consider a prototype partial differential operator of parabolic nature

$$
L u:=\vartheta \frac{\partial u}{\partial t}-\alpha \frac{\partial^{2} u}{\partial x^{2}}-\beta \frac{\partial u}{\partial x}-\gamma u
$$

We shall consider a very general situation that the coefficients are arbitrary functions in time $t$ and space $x$ on a general domain $D_{T, I}=(0, T) \times I, I$ is any interval.

We wish, for example, to construct some natural solution of

$$
\begin{aligned}
& L u(t, x)=g(t, x), \quad(t, x) \in(0, T) \times I, \\
& u(t, x)=0, \quad(t, x) \in(0, T) \times \partial I, \\
& u(0, x)=u_{0}(x), \quad x \in I,
\end{aligned}
$$

for very general functions $g$ and $u_{0}$ on a general domain $D_{T, I}$.

For a practical construction of the solution, we obtain the following formula (upon a finite number of data as it may be recognized in the statement).

Theorem 11. Let us fix a positive number $h$ and take a finite number of points $\left\{\left(t_{l}, x_{j}\right)\right\}_{l, j=1}^{n}$ of $D_{T, I}$ such that

$$
\left(\vartheta\left(t_{l}, x_{j}\right), \alpha\left(t_{l}, x_{j}\right), \beta\left(t_{l}, x_{j}\right), \gamma\left(t_{l}, x_{j}\right)\right) \neq 0
$$

as vectors, for each $(l, j)$. Then, the optimal solution $u_{h}^{A}$ of the equation (79) is given by

$$
u_{h}^{A}(t, x)=\frac{1}{(2 \pi)^{2}} \int_{D_{h}} F_{h}^{A}(\xi) e^{-i X \cdot \xi} d \xi \quad \text { for } X=(t, x)
$$

in terms of the function $F_{h}^{A} \in L_{2}\left(D_{h}\right)$, with $D_{h}=((-\pi / h,+\pi / h) \times(-\pi / h,+\pi / h))$ in the sense that $F_{h}^{A}$ has the minimum norm in $L_{2}\left(D_{h}\right)$ among the functions $F \in$ $L_{2}\left(D_{h}\right)$ satisfying: 


$$
\begin{aligned}
\frac{1}{(2 \pi)^{2}} \int_{\mathbb{R}^{2}} F(\xi)\left[\vartheta(t, x)\left(-i \xi_{1}\right)-\alpha(t, x)\left(-\xi_{2}^{2}\right)-\beta(t, x)\left(-i \xi_{2}\right)-\gamma(t, x)\right] \\
\cdot \chi_{D_{h}}(\xi) \exp (-i X \cdot \xi) d \xi=g(t, x),(80)
\end{aligned}
$$

where $\chi_{D_{h}}(\xi)$ denotes the characteristic function of $D_{h}$, and for all $X=X_{l j}$, i.e., $(t, x)=\left(t_{l}, x_{j}\right)$ and for the function space $L_{2}\left(D_{h}\right)$.

The best extremal function $F_{h}^{A}$ is given by

$$
\begin{aligned}
F_{h}^{A}(\xi)= & \sum_{l, j, l^{\prime}, j^{\prime}=1}^{n} g\left(t_{l}, x_{j}\right) \widetilde{a_{l j, l^{\prime}} j^{\prime}} \\
& \cdot\left(\vartheta\left(t_{l^{\prime}}, x_{j^{\prime}}\right)\left(-i \xi_{1}\right)-\alpha\left(t_{l^{\prime}}, x_{j^{\prime}}\right)\left(-\xi_{2}^{2}\right)-\beta\left(t_{l^{\prime}}, x_{j^{\prime}}\right)\left(-i \xi_{2}\right)-\gamma\left(t_{t^{\prime}}, x_{j^{\prime}}\right)\right) \\
& \cdot \exp \left(i X_{l^{\prime} j^{\prime}}, \xi\right) .
\end{aligned}
$$

We are considering in here the matrix $A=\left\{a_{\imath j, l^{\prime} j^{\prime}}\right\}\left(l, \iota^{\prime}, j, j^{\prime}=1, \ldots, n\right)$ formed by the elements

$$
a_{\imath j, l^{\prime} j^{\prime}}=K_{h h}\left(X_{l j}, X_{l^{\prime} j^{\prime}}\right)
$$

with

$$
\begin{aligned}
K_{h h}\left(X, X^{\prime}\right)= & \frac{1}{(2 \pi)^{2}} \int_{\mathbb{R}^{2}}\left[\vartheta(t, x)\left(-i \xi_{1}\right)-\alpha(t, x)\left(-\xi_{2}^{2}\right)-\beta(t, x)\left(-i \xi_{2}\right)-\gamma(t, x)\right] \\
& \cdot \frac{\left.\cdot \vartheta\left(t^{\prime}, x^{\prime}\right)\left(-i \xi_{1}\right)-\alpha\left(t^{\prime}, x^{\prime}\right)\left(-\xi_{2}^{2}\right)-\beta\left(t^{\prime}, x^{\prime}\right)\left(-i \xi_{2}\right)-\gamma\left(t^{\prime}, x^{\prime}\right)\right]}{} \\
& \left.\cdot \chi_{D_{h}}(\xi) \exp \left(-i\left(X-X^{\prime}\right) \cdot \xi\right)\right) d \xi
\end{aligned}
$$

which is positive definite and the $\widetilde{a_{i j, l^{\prime} j^{\prime}}}$ are the elements of the inverse of $\bar{A}$ (the complex conjugate of $A$ ).

Therefore, the optimal solution $u_{h}^{A}$ of the equation (79) is given by

$$
\begin{aligned}
u_{h}^{A}(t, x)= & \sum_{l, j, l^{\prime}, j^{\prime}=1}^{n} g\left(t_{l}, x_{j}\right) \widetilde{a_{l j, l^{\prime} j^{\prime}}} \\
& \frac{1}{(2 \pi)^{2}}\left[\overline{i \vartheta\left(t_{l^{\prime}}, x_{j^{\prime}}\right)} \int_{-\frac{\pi}{h}}^{\frac{\pi}{h}} \xi_{1} e^{-i\left(t-t_{t^{\prime}}\right) \xi_{1}} \int_{-\frac{\pi}{h}}^{\frac{\pi}{h}} e^{-i\left(x-x_{j^{\prime}}\right) \xi_{2}} d \xi_{2} d \xi_{1}\right. \\
& +\overline{\alpha\left(t_{t^{\prime}}, x_{j^{\prime}}\right)} \int_{-\frac{\pi}{h}}^{\frac{\pi}{h}} e^{-i\left(t-t_{\iota^{\prime}}\right) \xi_{1}} \int_{-\frac{\pi}{h}}^{\frac{\pi}{h}} \xi_{2}^{2} e^{-i\left(x-x_{j^{\prime}}\right) \xi_{2}} d \xi_{2} d \xi_{1} \\
& -i \overline{\beta\left(t_{l^{\prime}}, x_{j^{\prime}}\right)} \int_{-\frac{\pi}{h}}^{\frac{\pi}{h}} e^{-i\left(t-t_{t^{\prime}}\right) \xi_{1}} \int_{-\frac{\pi}{h}}^{\frac{\pi}{h}} \xi_{2} e^{-i\left(x-x_{j^{\prime}}\right) \xi_{2}} d \xi_{2} d \xi_{1} \\
& \left.-\overline{\gamma\left(t_{t^{\prime}}, x_{j^{\prime}}\right)} \int_{-\frac{\pi}{h}}^{\frac{\pi}{h}} e^{-i\left(t-t_{t^{\prime}}\right) \xi_{1}} \int_{-\frac{\pi}{h}}^{\frac{\pi}{h}} e^{-i\left(x-x_{j^{\prime}}\right) \xi_{2}} d \xi_{2} d \xi_{1}\right]
\end{aligned}
$$

In the next two results, we present the optimal solution for particular typical cases of equation (79). 
Corollary 3. If $\vartheta(t, x) \equiv 1, \alpha(t, x) \equiv 1, \beta(t, x) \equiv 0$ and $\gamma(t, x) \equiv 0$, then equation (79) reduces to an inhomogeneous heat equation and then the optimal solution of (79) is given as

$$
\begin{aligned}
u_{h}^{A}(t, x)= & \sum_{l, j, l^{\prime}, j^{\prime}=1}^{n} g\left(t_{l}, x_{j}\right) \widetilde{a_{l j, l^{\prime}} j^{\prime}} \frac{1}{(2 \pi)^{2}}\left[i \int_{-\frac{\pi}{h}}^{\frac{\pi}{h}} \xi_{1} e^{-i\left(t-t_{l^{\prime}}\right) \xi_{1}} \int_{-\frac{\pi}{h}}^{\frac{\pi}{h}} e^{-i\left(x-x_{j^{\prime}}\right) \xi_{2}} d \xi_{2} d \xi_{1}\right. \\
& \left.+\int_{-\frac{\pi}{h}}^{\frac{\pi}{h}} e^{-i\left(t-t_{t^{\prime}}\right) \xi_{1}} \int_{-\frac{\pi}{h}}^{\frac{\pi}{h}} \xi_{2}^{2} e^{-i\left(x-x_{j^{\prime}}\right) \xi_{2}} d \xi_{2} d \xi_{1}\right] .
\end{aligned}
$$

Corollary 4. If $\vartheta(t, x) \equiv 1, \alpha(t, x) \equiv \frac{i \hbar}{2 m}, \beta(t, x) \equiv 0$ and $\gamma(t, x) \equiv 0$, then equation (79) reduces to an inhomogeneous Schrödinger equation and then the optimal solution of (79) is given as

$$
\begin{aligned}
u_{h}^{A}(t, x)= & \sum_{l, j, l^{\prime}, j^{\prime}=1}^{n} g\left(t_{l}, x_{j}\right) \widetilde{a_{l j, l^{\prime}} j^{\prime}} \frac{1}{(2 \pi)^{2}}\left[i \int_{-\frac{\pi}{h}}^{\frac{\pi}{h}} \xi_{1} e^{-i\left(t-t_{l^{\prime}}\right) \xi_{1}} \int_{-\frac{\pi}{h}}^{\frac{\pi}{h}} e^{-i\left(x-x_{j^{\prime}}\right) \xi_{2}} d \xi_{2} d \xi_{1}\right. \\
& \left.-\frac{i \hbar}{2 m} \int_{-\frac{\pi}{h}}^{\frac{\pi}{h}} e^{-i\left(t-t_{l^{\prime}}\right) \xi_{1}} \int_{-\frac{\pi}{h}}^{\frac{\pi}{h}} \xi_{2}^{2} e^{-i\left(x-x_{j^{\prime}}\right) \xi_{2}} d \xi_{2} d \xi_{1}\right] .
\end{aligned}
$$

Here, we assume that equation (79) is valid on $D_{T, I}$ and so, as some practical case we would like to consider the equation in (79) on $D_{T, I}$ satisfying some initial and boundary conditions. In the present case, the boundary conditions are given as zero at infinity for $I=\mathbb{R}$ by the property of the Paley-Wiener spaces.

Since the functions in the Paley-Wiener spaces are decreasing exponentially, for the zero boundary condition solutions, for a large interval $I$, Theorem 11 will give good approximate solutions. If $h$ is smaller, then we can consider the interval $I$ as a more smaller one.

However, our result gives the approximate general solutions satisfying initial and boundary conditions. For example, for a finite interval $I=(a, b)$, we consider $t_{1}=0$ and

$$
\vartheta\left(0, x_{j}\right)=\alpha\left(0, x_{j}\right)=\beta\left(0, x_{j}\right)=0,
$$

then $\gamma\left(0, x_{j}\right) \neq 0$ and we have

$$
\gamma\left(0, x_{j}\right) u\left(0, x_{j}\right)=g\left(0, x_{j}\right) \Rightarrow u_{0}\left(x_{j}\right)=\frac{g\left(0, x_{j}\right)}{\gamma\left(0, x_{j}\right)} .
$$

Then, we see that we can consider the initial condition which may be given, in a very general situation.

We will now turn to a different type of equations. Anyway, once again, the argument presented in here will show that our results will continue valid in a very general situation. However, in order to state the results and methods in a simpler manner, we shall consider the following two dimensional second-order differential operator of Laplace type: 


$$
L u:=\alpha \frac{\partial^{2} u}{\partial x^{2}}+\beta \frac{\partial^{2} u}{\partial y^{2}}
$$

where $I$ is any open interval.

The main goal is to construct some natural solution of

$$
L u(x, y)=g(x, y), \quad(x, y) \in I \times I,
$$

satisfying the boundary condition

$$
u(x, y)=u_{f}(x, y), \quad(x, y) \in \partial I \times \partial I,
$$

where the function $u_{f}$ is defined on the boundary of $I \times I$ and such that

$$
u_{f} \rightarrow 0 \quad \text { as } \quad|X| \rightarrow \infty,
$$

with $X=(x, y)$.

For a practical construction of the solution, we obtain the following formula (once again by considering the technique of taking a finite number of data as it may be recognized in the statement).

Theorem 12. Let us fix a positive number $h$ and take a finite number of points $\left\{\left(x_{l}, y_{j}\right)\right\}_{l, j=1}^{n}$ of $D_{I, I}$ such that

$$
\left(\alpha\left(x_{l}, y_{j}\right), \beta\left(x_{l}, y_{j}\right)\right) \neq 0
$$

as vectors, for each $\left(x_{l}, y_{j}\right)$. Then, the optimal solution $u_{h}^{A}$ of the equation (84) is given by, for $X=(x, y)$,

$$
u_{h}^{A}(x, y)=\frac{1}{(2 \pi)^{2}} \int_{D_{h}} F_{h}^{A}(\xi) e^{-i X \cdot \xi} d \xi
$$

in terms of the function $F_{h}^{A} \in L_{2}\left(D_{h}\right), D_{h}=((-\pi / h,+\pi / h) \times(-\pi / h,+\pi / h))$ in the sense that $F_{h}^{A}$ has the minimum norm in $L_{2}\left(D_{h}\right)$ among the functions $F \in L_{2}\left(D_{h}\right)$ satisfying:

$$
\left.\frac{1}{(2 \pi)^{2}} \int_{\mathbb{R}^{2}} F(\xi)\left[\alpha(x, y) \xi_{1}^{2}+\beta(x, y) \xi_{2}^{2}\right)\right] \chi_{D_{h}}(\xi) \exp (-i X \cdot \xi) d \xi+g(x, y)=0
$$

for all $X=X_{l j}$, i.e., $(x, y)=\left(x_{l}, y_{j}\right)$ and for the function space $L_{2}\left(D_{h}\right)$.

The best extremal function $F_{h}^{A}$ is given by

$$
F_{h}^{A}(\xi)=-\sum_{l, j, l^{\prime}, j^{\prime}=1}^{n} g\left(t_{l}, x_{j}\right) \widetilde{a_{l j, l^{\prime} j^{\prime}}} \overline{\left(\alpha\left(x_{l^{\prime}}, y_{j^{\prime}}\right) \xi_{1}^{2}+\beta\left(x_{l^{\prime}}, y_{j^{\prime}}\right) \xi_{2}^{2}\right)} \exp \left(i X_{l^{\prime} j^{\prime}} \cdot \xi\right) .
$$

Moreover, the matrix $A=\left\{a_{\imath j, l^{\prime} j^{\prime}}\right\}\left(l, l^{\prime}, j, j^{\prime}=1, \ldots, n\right)$ formed by the elements

$$
a_{l j, l^{\prime} j^{\prime}}=K_{h h}\left(X_{l j}, X_{l^{\prime} j^{\prime}}\right)
$$


with

$$
\begin{aligned}
K_{h h}\left(X, X^{\prime}\right)= & \frac{1}{(2 \pi)^{2}} \int_{\mathbb{R}^{2}}\left(\alpha(x, y) \xi_{1}^{2}+\beta(x, y) \xi_{2}^{2}\right) \overline{\left(\alpha\left(x^{\prime}, y^{\prime}\right) \xi_{1}^{2}+\beta\left(x^{\prime}, y^{\prime}\right) \xi_{2}^{2}\right)} \\
& \left.\cdot \chi_{D_{h}}(\xi) \exp \left(-i\left(X-X^{\prime}\right) \cdot \xi\right)\right) d \xi
\end{aligned}
$$

which is positive definite and we are using above the elements $\widetilde{a_{l j, l^{\prime} j^{\prime}}}$ which are the entries of the inverse of $\bar{A}$ (the complex conjugate of $A$ ).

Therefore, the optimal solution $u_{h}^{A}$ of the equation (84) is given by

$$
\begin{aligned}
u_{h}^{A}(x, y)= & -\sum_{l, j, l^{\prime}, j^{\prime}=1}^{n} g\left(x_{l}, y_{j}\right) \widetilde{a_{l j, l^{\prime} j^{\prime}}} \frac{1}{(2 \pi)^{2}} \\
& \cdot\left[\overline{\alpha\left(x_{l^{\prime}}, y_{j^{\prime}}\right)} \int_{-\frac{\pi}{h}}^{\frac{\pi}{h}} \xi_{1}^{2} e^{-i\left(x-x_{l^{\prime}}\right) \xi_{1}} \int_{-\frac{\pi}{h}}^{\frac{\pi}{h}} e^{-i\left(y-y_{j^{\prime}}\right) \xi_{2}} d \xi_{2} d \xi_{1}\right. \\
& \left.+\overline{\beta\left(x_{l^{\prime}}, y_{j^{\prime}}\right)} \int_{-\frac{\pi}{h}}^{\frac{\pi}{h}} e^{-i\left(x-x_{l^{\prime}}\right) \xi_{1}} \int_{-\frac{\pi}{h}}^{\frac{\pi}{h}} \xi_{2}^{2} e^{-i\left(y-y_{j^{\prime}}\right) \xi_{2}} d \xi_{2} d \xi_{1}\right] .
\end{aligned}
$$

In the next two results, we present the optimal solution for the particular typical case of equation (84).

Corollary 5. If $\alpha(t, x) \equiv 1$ and $\beta(t, x) \equiv 1$, then equation (84) reduces to an inhomogeneous Poisson equation and then the optimal solution of (84) is given as

$$
\begin{aligned}
u_{h}^{A}(x, y)= & -\sum_{l, j, l^{\prime}, j^{\prime}=1}^{n} g\left(x_{l}, y_{j}\right) \widetilde{a_{l j, l^{\prime} j^{\prime}}} \frac{1}{(2 \pi)^{2}} \\
& {\left[\int_{-\frac{\pi}{h}}^{\frac{\pi}{h}} \xi_{1}^{2} e^{-i\left(x-x_{l^{\prime}}\right) \xi_{1}} \int_{-\frac{\pi}{h}}^{\frac{\pi}{h}} e^{-i\left(y-y_{j^{\prime}}\right) \xi_{2}} d \xi_{2} d \xi_{1}\right.} \\
& \left.+\int_{-\frac{\pi}{h}}^{\frac{\pi}{h}} e^{-i\left(x-x_{l^{\prime}}\right) \xi_{1}} \int_{-\frac{\pi}{h}}^{\frac{\pi}{h}} \xi_{2}^{2} e^{-i\left(y-y_{j^{\prime}}\right) \xi_{2}} d \xi_{2} d \xi_{1}\right] .
\end{aligned}
$$

Corollary 6. If $\alpha(t, x) \equiv y$ and $\beta(t, x) \equiv 1$, then equation (84) reduces to an inhomogeneous Tricomi equation and then the optimal solution of (84) is given as

$$
\begin{aligned}
u_{h}^{A}(x, y)= & -\sum_{l, j, l^{\prime}, j^{\prime}=1}^{n} g\left(x_{l}, y_{j}\right) \widetilde{a_{l j, l^{\prime} j^{\prime}}} \frac{1}{(2 \pi)^{2}} \\
& {\left[\overline{y_{j^{\prime}}} \int_{-\frac{\pi}{h}}^{\frac{\pi}{h}} \xi_{1}^{2} e^{-i\left(x-x_{l^{\prime}}\right) \xi_{1}} \int_{-\frac{\pi}{h}}^{\frac{\pi}{h}} e^{-i\left(y-y_{j^{\prime}}\right) \xi_{2}} d \xi_{2} d \xi_{1}\right.} \\
& \left.+\int_{-\frac{\pi}{h}}^{\frac{\pi}{h}} e^{-i\left(x-x_{l^{\prime}}\right) \xi_{1}} \int_{-\frac{\pi}{h}}^{\frac{\pi}{h}} \xi_{2}^{2} e^{-i\left(y-y_{j^{\prime}}\right) \xi_{2}} d \xi_{2} d \xi_{1}\right] .
\end{aligned}
$$

Note that, the Tricomi equation is of mixed type and has been associated with transonic flow, where $u$ may be thought of as a stream function or a perturbation velocity potential, when considering a source $g$ and boundary data $u_{f}$ to be specified. 
The equation is elliptic for $y>0$, hyperbolic for $y<0$, and parabolic for $y=0$, an interface called the sonic line as it separates regions of subsonic and supersonic flow. It is well-known that the equation (84) yields a well-posed problem if $u_{f}$ is prescribed only on suitable portions of the boundary - a study initiated by Tricomi.

\subsection{First Order Partial Differential Equations}

In this section we will consider a general two dimensional first-order (hypoelliptic) differential operator,

$$
L u:=\alpha(x, y) \frac{\partial u}{\partial x}+\beta(x, y) \frac{\partial u}{\partial y}
$$

The idea is to implement our method so that some natural solution of

$$
L u(x, y)=g(x, y), \text { on } \mathbb{R}^{2} .
$$

will be constructed.

Theorem 13. Let us fix a positive number $h$ and take a finite number of points $\left\{\left(x_{l}, y_{j}\right)\right\}_{l, j=1}^{n}$ of $\mathbb{R}^{2}$ such that

$$
\left(\alpha\left(x_{l}, y_{j}\right), \beta\left(x_{l}, y_{j}\right)\right) \neq 0
$$

as vectors. Then, the optimal solution $u_{h}^{A}$ of the equation (88) is given by

$$
u_{h}^{A}(x, y)=\frac{1}{(2 \pi)^{2}} \int_{D_{h}} F_{h}^{A}(\xi) e^{-i X \cdot \xi} d \xi \quad \text { for } X=(x, y)
$$

in terms of the function $F_{h}^{A} \in L_{2}\left(D_{h}\right), D_{h}=((-\pi / h,+\pi / h) \times(-\pi / h,+\pi / h))$ in the sense that $F_{h}^{A}$ has the minimum norm in $L_{2}\left(D_{h}\right)$ among all the functions $F \in L_{2}\left(D_{h}\right)$ satisfying

$$
\left.\frac{1}{(2 \pi)^{2}} \int_{\mathbb{R}^{2}} F(\xi)\left[-i \alpha(x, y) \xi_{1}-i \beta(x, y) \xi_{2}\right)\right] \chi_{D_{h}}(\xi) \exp (-i X \cdot \xi) d \xi=g(x, y)
$$

for all $X=X_{l j}$, i.e., $(x, y)=\left(x_{l}, y_{j}\right)$ and for the function space $L_{2}\left(D_{h}\right)$.

The best extremal function $F_{h}^{A}$ is given by

$$
F_{h}^{A}(\xi)=\sum_{l, j, l^{\prime}, j^{\prime}=1}^{n} g\left(t_{l}, x_{j}\right) \widetilde{a_{l j, l^{\prime}} j^{\prime}}\left[\overline{\left.-i \alpha\left(x_{l^{\prime}}, y_{j^{\prime}}\right) \xi_{1}-i \beta\left(x_{l^{\prime}}, y_{j^{\prime}}\right) \xi_{2}\right)}\right] \exp \left(i X_{l^{\prime} j^{\prime}} \cdot \xi\right)
$$

where $\widetilde{a_{l j, l^{\prime} j^{\prime}}}$ are the entries of the inverse of $\bar{A}$, the complex conjugate of the matrix $A=\left\{a_{l j, l^{\prime} j^{\prime}}\right\}\left(l, l^{\prime}, j, j^{\prime}=1, \ldots, n\right)$ formed by the elements 


$$
a_{\imath j, l^{\prime} j^{\prime}}=K_{h h}\left(X_{\imath j}, X_{l^{\prime} j^{\prime}}\right)
$$

with

$$
\begin{aligned}
K_{h h}\left(X, X^{\prime}\right)= & \left.\frac{1}{(2 \pi)^{2}} \int_{\mathbb{R}^{2}}\left[-i \alpha(x, y) \xi_{1}-i \beta(x, y) \xi_{2}\right)\right] \\
& {\left.\left[\overline{\left(-i \alpha\left(x^{\prime}, y^{\prime}\right) \xi_{1}-i \beta\left(x^{\prime}, y^{\prime}\right) \xi_{2}\right)}\right] \chi_{D_{h}}(\xi) \exp \left(-i\left(X-X^{\prime}\right) \cdot \xi\right)\right) d \xi }
\end{aligned}
$$

which is positive definite.

Therefore, the optimal solution $u_{h}^{A}$ of the equation (88) is given by

$$
\begin{aligned}
u_{h}^{A}(x, y)= & \sum_{l, j, l^{\prime}, j^{\prime}=1}^{n} g\left(x_{l}, y_{j}\right) \widetilde{a_{l j, l^{\prime} j^{\prime}}} \frac{1}{(2 \pi)^{2}} \\
& \cdot\left[\overline{i \alpha\left(x_{l^{\prime}}, y_{j^{\prime}}\right)} \int_{-\frac{\pi}{h}}^{\frac{\pi}{h}} \xi_{1} e^{-i\left(x-x_{l^{\prime}}\right) \xi_{1}} \int_{-\frac{\pi}{h}}^{\frac{\pi}{h}} e^{-i\left(y-y_{j^{\prime}}\right) \xi_{2}} d \xi_{2} d \xi_{1}\right. \\
& \left.+i \overline{\beta\left(x_{l^{\prime}}, y_{j^{\prime}}\right)} \int_{-\frac{\pi}{h}}^{\frac{\pi}{h}} e^{-i\left(x-x_{\iota^{\prime}}\right) \xi_{1}} \int_{-\frac{\pi}{h}}^{\frac{\pi}{h}} \xi_{2} e^{-i\left(y-y_{j^{\prime}}\right) \xi_{2}} d \xi_{2} d \xi_{1}\right]
\end{aligned}
$$

In the next result, we present the optimal solution for a particular case of equation (88).

Corollary 7. If $\alpha(x, y)=-1+2 i(x+y)$ and $\beta(x, y)=-i+2(x+y)$, then equation (88) reduces to an inhomogeneous, i.e.

$$
-\frac{\partial u}{\partial x}-i \frac{\partial u}{\partial y}+2 i(x+y) \frac{\partial u}{\partial z}=g(x, y),
$$

with $\frac{\partial}{\partial z}$ a complex differential operator and then the optimal solution is given as

$$
\begin{aligned}
u_{h}^{A}(x, y)= & \sum_{l, j, l^{\prime}, j^{\prime}=1}^{n} g\left(x_{l}, y_{j}\right) \widetilde{a_{l j, l^{\prime} j^{\prime}}} \frac{1}{(2 \pi)^{2}} \\
& \cdot\left[\left(-i+2 x_{\iota^{\prime}}+2 y_{j^{\prime}}\right) \int_{-\frac{\pi}{h}}^{\frac{\pi}{h}} \xi_{1} e^{-i\left(x-x_{\iota^{\prime}}\right) \xi_{1}} \int_{-\frac{\pi}{h}}^{\frac{\pi}{h}} e^{-i\left(y-y_{j^{\prime}}\right) \xi_{2}} d \xi_{2} d \xi_{1}\right. \\
& \left.+\left(-1+2 i x_{\iota^{\prime}}+2 i y_{j^{\prime}}\right) \int_{-\frac{\pi}{h}}^{\frac{\pi}{h}} e^{-i\left(x-x_{\iota^{\prime}}\right) \xi_{1}} \int_{-\frac{\pi}{h}}^{\frac{\pi}{h}} \xi_{2} e^{-i\left(y-y_{j^{\prime}}\right) \xi_{2}} d \xi_{2} d \xi_{1}\right]
\end{aligned}
$$

\subsection{Existence Problem of the Differential Equation}

Following (I) and (II) in Proposition 1, we shall first consider the existence of the solution of the previous differential equations. For the image identification of the in- 
tegral transform $((72),(80),(85),(89))$, from the corresponding representation $((10)$, (82), (86), (90)), we must realize the reproducing kernel Hilbert space $H_{K_{h h}}$ admitting the reproducing kernel $K_{h h}\left(X, X^{\prime}\right)$. However, we know that if ((10), (82), (86), (90)) are not calculated in a good way, in general, its realization is very complicated and the inversion of the integral transform ((72), (80), (85), (89)) are involved. So, we shall consider the existence problem with a numerical treatment.

Following a general approach of the theory of reproducing kernels, we shall consider what in our opinion is a very natural and general method.

First, let us recall several facts and some notation. For two positive semi-definite matrices $K^{(1)}(p, q)$ and $K^{(2)}(p, q)$ on $E$, if $K^{(2)}(p, q)-K^{(1)}(p, q)$ is a positive semidefinite matrix on $E$, then we shall write it as follows:

$$
K^{(1)} \ll K^{(2)} .
$$

We see that the symbol $\ll$ is a partial ordering in the class of all positive semidefinite matrices on $E$ and, moreover, the following result holds.

Proposition 8. For two positive semi-definite matrices $K^{(1)}(p, q)$ and $K^{(2)}(p, q)$ on E,

$$
H_{K^{(1)}} \subseteq H_{K^{(2)}} \quad(\text { as } \text { members })
$$

if and only if there exists a positive constant $\Gamma$ such that

$$
K^{(1)}(p, q) \ll \Gamma^{2} K^{(2)}(p, q) .
$$

Here, the minimum of such constants $\Gamma$ coincides with the norm of the inclusion map $J$ from $H_{K^{(1)}}$ into $H_{K^{(2)}}$.

By using this Proposition 8, we can give completely the existence condition of the solutions as follows:

Theorem 14. For any function $g$ there exists a solution $u(x, y) \in W\left(\frac{\pi}{h}\right)$ satisfying (71), (79), (84), (88) if and only if for some constant $\Gamma$

$$
g(x, y) \overline{g\left(x^{\prime}, y^{\prime}\right)} \ll \Gamma^{2} K_{h h}\left(X, X^{\prime}\right) ;
$$

that is, for any finite number of points $\left\{\left(t_{l}, x_{j}\right)\right\}$ and for any constants $\left\{C_{l j}\right\}$,

$$
\left|\sum_{l, j=1}^{n} C_{l j} g\left(t_{l}, x_{j}\right)\right|^{2} \leq \Gamma^{2} \sum_{l, j, l^{\prime}, j^{\prime}=1}^{n} C_{l j} \overline{C_{l^{\prime} j^{\prime}}} K_{h h}\left(X_{l j}, X_{l^{\prime} j^{\prime}}\right)
$$

holds with $X_{l j}=\left(x_{l}, y_{j}\right), X_{l^{\prime} j^{\prime}}=\left(x_{l^{\prime}}, y_{j^{\prime}}\right)$. If this is the case, then the norm of $g$ in $H_{K_{h h}}$ is given by the infimum $\Gamma$ satisfying those inequalities.

At this moment, we cannot provide any further information about the uniqueness of the solutions. 


\section{Convolution Integral Equations}

Let us now introduce the function spaces $\mathscr{F}\left(\rho_{j}\right)(j=1,2,3)$, where $\rho_{j}$ are nonnegative and integrable functions on $\mathbb{R}$. Each of these spaces collects all elements $F_{j}$ which satisfy

$$
\int \frac{\left|F_{j}(t)\right|^{2}}{\rho_{j}(t)} d t<\infty
$$

on the support of $\rho_{j}$, and are simply $F_{j}=0$ on the outside of the support of $\rho_{j}$. Additionally, we also set

$$
\Omega\left(t ; \rho_{1}, \rho_{2}, \rho_{3}\right):=\rho_{1} *\left(2 \pi+\rho_{2}+\rho_{3}\right)
$$

(for the usual convolution $*$ ).

In particular, note that all the functions $F_{j} \in \mathscr{F}\left(\rho_{j}\right)(j=1,2,3)$ belong to $L_{1}(\mathbb{R})$, as we see from the Schwarz inequality.

We set

$$
f_{j}(x)=\left(L_{j} F_{j}\right)(x)=\frac{1}{2 \pi} \int_{\mathbb{R}} F_{j}(t) \exp (i t \cdot x) d t
$$

and

$$
g(x)=\frac{1}{(2 \pi)^{2}} \int_{\mathbb{R}} G(t) \exp (i x \cdot t) d t .
$$

Theorem 15. Let $\rho_{j}(j=1,2,3)$ be non-negative and integrable functions on $\mathbb{R}$ which allow us to consider the just introduced spaces $\mathscr{F}\left(\rho_{j}\right)$.

For any fixed $F_{j} \in \mathscr{F}\left(\rho_{j}\right), j=2,3$ (with $\alpha+F_{2}+F_{3}$ not zero on a positive measure set), and for a function $G$ satisfying

$$
\int_{\mathbb{R}}|G(t)|^{2} \Omega\left(t ; \rho_{1}, \rho_{2}, \rho_{3}\right)^{-1} d t<\infty
$$

we consider the integral equation

$$
2 \pi \alpha F_{1}(t)+\int_{\mathbb{R}} F_{1}(\xi) F_{2}(t-\xi) d \xi+\int_{\mathbb{R}} F_{1}(\xi) F_{3}(t-\xi) d \xi=G(t) .
$$

When the function $\alpha+f_{2}(x)+f_{3}(x)$ is not vanishing, for any function $g$ satisfying the condition

$$
\int_{\mathbb{R}}\left|\int_{\mathbb{R}} \frac{g(x)}{\alpha+f_{2}(x)+f_{3}(x)}\right|^{2} \frac{d t}{\rho_{1}(t)},
$$

there exists a uniquely determined solution $F_{1}$ belonging to $\mathscr{F}\left(\rho_{1}\right)$ and the solution $F_{1}$ is represented by

$$
F_{1}(t)=\frac{1}{2 \pi} \int_{\mathbb{R}} \frac{g(x)}{\alpha+f_{2}(x)+f_{3}(x)} \exp (-i t \cdot x) d x .
$$

In this Theorem 15, we can see that the function space where $g$ belongs to is a very natural one for considering in the integral equation (97) together with the other 
function spaces for the integral kernels and solutions, from the theory of reproducing kernels.

The criteria (96) will be, in general, involved and so, we, in general, proposed numerical calculation algorithms of some general fractional functions by using the Tikhonov regularization and the theory of reproducing kernels; cf. [8].

For some practical construction of the solution, we obtain the following formula.

Theorem 16. Assume that $\alpha+f_{2}(x)+f_{3}(x)$ is not zero on a positive measure set. We take a finite number of points $\left\{p_{j}\right\}_{j=1}^{n}$ satisfying that $\alpha+f_{2}\left(p_{j}\right)+f_{3}\left(p_{j}\right)$ is not zero, for any $j$.

Then, the optimal solution $F_{1}^{A}$ of the equation (95) satisfying that, for the equation

$$
\alpha f_{1}(x)+f_{1}(x) f_{2}(x)+f_{1}(x) f_{3}(x)=g(x),
$$

which is transformed to the integral equation (95) by the Fourier integrals, and for all $j$,

$$
\alpha f_{1}\left(p_{j}\right)+f_{1}\left(p_{j}\right) f_{2}\left(p_{j}\right)+f_{1}\left(p_{j}\right) f_{3}\left(p_{j}\right)=g\left(p_{j}\right)
$$

is given by

$$
F_{1}^{A}(t)=\sum_{j, j^{\prime}=1}^{n} g\left(p_{j}\right) \widetilde{a_{j j^{\prime}}} \boldsymbol{\aleph}\left(p_{j^{\prime}}, t\right) .
$$

Here, the matrix A formed by the elements

$$
a_{j j^{\prime}}=K_{\varphi}\left(p_{j}, p_{j^{\prime}}\right)
$$

with

$$
K_{\varphi}(x, y)=K_{1}(x, y)\left(\alpha+f_{2}(x)+f_{3}(x)\right) \overline{\left(\alpha+f_{2}(y)+f_{3}(y)\right)}
$$

and where

$$
K_{1}(x, y)=\frac{1}{2 \pi} \int_{\mathbb{R}} \exp (i(x-y) \cdot t) \rho_{1}(t) d t
$$

is positive definite and $\widetilde{a_{j j^{\prime}}}$ are the elements of the inverse of $\bar{A}$ (the complex conjugate of $A)$. Further, $\boldsymbol{\aleph}\left(p_{j^{\prime}}, t\right)$ are given by

$$
\exp \left(-i p_{j^{\prime}} \cdot t\right) \overline{\left(\alpha+f_{2}\left(p_{j^{\prime}}\right)+f_{3}\left(p_{j^{\prime}}\right)\right)}
$$

The optimal solution means that it minimizes the norm squares

$$
\frac{1}{2 \pi} \int_{\mathbb{R}}\left|F_{1}(t)\right|^{2} \rho_{1}(t)^{-1} d t
$$

among the functions $F_{1}$ satisfying the above conditions.

The point value estimation of the approximate solution $F_{1}^{A}(t)$ is given in terms of the points $\left\{p_{j}\right\}_{j=1}^{n}$ by

$$
\left|F_{1}^{A}(t)\right| \leq\left(\sum_{j, j^{\prime}=1}^{n} g\left(p_{j}\right) \widetilde{a_{j j^{\prime}}} \overline{g\left(p_{j^{\prime}}\right)}\right)^{1 / 2} \cdot\left(\sum_{j, j^{\prime}=1}^{n} \aleph\left(p_{j}, t\right) \widetilde{a_{j j^{\prime}}} \overline{\aleph\left(p_{j^{\prime}}, t\right)}\right)^{1 / 2}
$$


In particular, note that for the approximate solution (100), we do not use the values $\left\{f_{1}\left(p_{j}\right)\right\}$; this means that we do not solve the algebraic equations (99). This point of view will be very interesting and important for the formula (100).

\section{Singular Integral Equations}

Singular integral equations arise in a great variety of problems which are useful for modeling a wide range of applied problems (e.g., in acoustics, elasticity, fluid dynamics, radiative equilibrium and fracture mechanics - just to cite a few subareas of applied sciences). Despite a great variety of existing methods, singular integral equations are typically difficult to solve analytically and therefore additional knowledge about their approximate solutions is still needed. Anyway, many numerical methods have been developed already for such type of equations. Some of those are known as the method of interpolatory quadrature rules based on piecewise polynomials, Legendre polynomial method, Bernstein polynomial method, Taylorseries expansion method, Galerkin method and collocation methods. However, these methods typically require a lot of computational effort in several steps of their approximation techniques. See the original paper [10] for the detailed references and survey for the singular integral equations.

In here, we will apply our technique to the following Carleman singular integral equation over a general interval, and having very general real or complex valued functions $a$ and $b$ as coefficients:

$$
(L y)(t):=a(t) y(t)+\frac{b(t)}{\pi i} \text { p.v. } \int_{\mathbb{R}} \frac{y(\zeta)}{\zeta-t} \mathrm{~d} \zeta=g(t), \quad t \in \mathscr{I},
$$

where $g$ is a given very general function, and $\mathscr{I}$ is a general interval on the real line. As mentioned, we shall call (101) an equation of Carleman type because it was discussed by T. Carleman, who first gave an explicit solution of this equation in closed form (when considering some conditions on the coefficients).

In case of $a^{2}(t)-b^{2}(t) \neq 0$, on $\mathscr{I}$, the operator $L$ is called a regular type operator (see [27]). An explicit solution of the equation (101) for a regular type operator (in convenient spaces of functions) is well-known. However, when $a^{2}(t)-b^{2}(t)=0$ (for $t$ in the integrability domain), there are solutions if and only if $g$ satisfies a special condition. The analysis of this case is important e.g. for the kinked crack problem (see, e.g., [16]). Accordingly, we shall exhibit here the application of our method to this equation which will give simple and natural approximate solutions for linear singular integral equations including the case where the condition of a regular type operator is violated. We will deal with a surprisingly general linear singular integral equation by our new discretization method.

Theorem 17. We fix a positive number $h$ and take a finite number of points $\left\{t_{j}\right\}_{j=1}^{n}$ of $\mathscr{I}$ satisfying that 


$$
\left\{\left[a\left(t_{j}\right)+b\left(t_{j}\right) \operatorname{sgn}(\xi)\right] \exp \left(-i t_{j} \xi\right)\right\}
$$

are linearly independent in the space $L_{2}(-\pi / h,+\pi / h)$.

Then, the optimal solution $y_{h}^{A}$ of the equation (101) has the form

$$
y_{h}^{A}(t)=\frac{1}{2 \pi} \int_{-\pi / h}^{\pi / h} F_{h}^{A}(\xi) e^{-i t \xi} d \xi
$$

satisfying $F_{h}^{A} \in L_{2}(-\pi / h,+\pi / h)$ in the sense that $F_{h}^{A}$ has the minimum norm in $L_{2}(-\pi / h,+\pi / h)$ among the functions $F \in L_{2}(-\pi / h,+\pi / h)$ satisfying, for the characteristic function $\chi_{h}(t)$ of the interval $(-\pi / h,+\pi / h)$ :

$$
a(t)\left(\mathscr{F}^{-1}\left(F \chi_{h}\right)\right)(t)+\frac{b(t)}{i}(-1)\left(\mathscr{H}^{F} \mathscr{F}^{-1}\left(F \chi_{h}\right)\right)(t)=2 \pi g(t)
$$

for all $t=t_{j}$. Here, we are using the Fourier transform $\mathscr{F}$ given by

$$
\mathscr{F}[f](\xi)=\frac{1}{\sqrt{2 \pi}} \int_{-\infty}^{\infty} f(t) e^{i \xi t} \mathrm{~d} t
$$

and the Hilbert transform $\mathscr{H}$,

$$
[\mathscr{H} y](t)=\frac{1}{\pi} p \cdot v \cdot \int_{-\infty}^{\infty} \frac{y(\zeta)}{t-\zeta} \mathrm{d} \zeta .
$$

The best extremal function $F_{h}^{A}$ is given by

$$
F_{h}^{A}(\xi)=\sum_{j, j^{\prime}=1}^{n} g\left(t_{j}\right) \widetilde{a_{j j^{\prime}}} \overline{\left(a\left(t_{j^{\prime}}\right)+b\left(t_{j^{\prime}}\right) \operatorname{sgn}(\xi)\right)} \exp \left(i t_{j^{\prime}} \cdot \xi\right) .
$$

Here, the matrix A formed by the elements

$$
a_{j j^{\prime}}=K_{h h}\left(t_{j}, t_{j^{\prime}}\right)
$$

with

$K_{h h}\left(t, t^{\prime}\right)=\frac{1}{2 \pi} \int_{\mathbb{R}}[a(t)+b(t) \operatorname{sgn}(\xi)] \overline{\left[a\left(t^{\prime}\right)+b\left(t^{\prime}\right) \operatorname{sgn}(\xi)\right]} \chi_{h}(\xi) \exp \left(-i\left(t-t^{\prime}\right) \xi\right) d \xi$

which is positive definite and $\widetilde{a_{j j^{\prime}}}$ are the elements of the inverse of $\bar{A}$ (the complex conjugate of $A$ ).

Therefore, the optimal solution $y_{h}^{A}$ of the equation (101) is given by

$$
\begin{aligned}
y_{h}^{A}(t)= & \sum_{j, j^{\prime}=1}^{n} g\left(t_{j}\right) \widetilde{a_{j j^{\prime}}}\left(\left(\overline{a\left(t_{j^{\prime}}\right)} \frac{1}{\pi\left(t-t_{j^{\prime}}\right)} \sin \frac{\pi}{h}\left(t-t_{j^{\prime}}\right)\right.\right. \\
& \left.+\overline{b\left(t_{j^{\prime}}\right)} \frac{1}{\pi i\left(t-t_{j^{\prime}}\right)}\left(1-\exp \left(-\frac{\pi}{h}\left(t-t_{j^{\prime}}\right) i\right)\right)\right) .
\end{aligned}
$$


In particular, note that for the approximate solution of $y_{h}^{A}$, we do not use the values $\left(\mathscr{F}^{-1}\left(F \chi_{h}\right)\right)\left(t_{j}\right)$. This means that we do not need to solve the algebraic equations (102). This point of view will be very interesting and important for obtaining the optimal and approximate solution of (101).

Furthermore, for the coefficients $a$ and $b$, they are arbitrary functions in their nature. Note that we do not assume their measurable property, even.

\section{Numerical Examples Associated with Theorem 9}

We shall show some numerical experiments of the proposed method for ordinary differential equations. In the following, we set $h=1$; we seek our solution in the Paley-Wiener space $W(\pi)$ with equi-spaced collocation points.

Example 4. We consider an ordinary differential equation

$$
y^{\prime \prime}(t)=4, \quad-1 \leq t \leq 1,
$$

without any boundary conditions. Equi-spaced points $\left\{t_{j}\right\}$ in the interval $[-1,1]$ are employed as the collocation points; more precisely, $t_{j}=-1+2(j-1) /(n-1), j=$ $1,2, \ldots, n$ for some positive number $n$.

Fig. 3 shows numerical results with 100 digits precision computation by multipleprecision arithmetic environment exflib [17], which provides arbitrarily accurate approximation of real numbers and their arithmetic on digital computers.

Here, we give a remark that optimality of the solution given in Theorem 9 is not $L_{2}$-norm minimality. The general solution is $y(t)=2 t^{2}+a t+b, a, b \in \mathbb{C}$, and the minimum norm solution in $L_{2}(-1,1)$ is $y_{0}(t)=2 t^{2}-2 / 3$. On the other hand, numerical solutions shown in Fig. 3 depend on $n$, and none of them approximate the minimum norm solution $y_{0}(t)$. However, note that they are different only by a shift because in here we do not impose boundary conditions.

Example 5. We consider a boundary value problem

$$
y^{\prime \prime}(t)=4 \quad(-1<t<1), \quad y(-1)=1, \quad y(1)=3,
$$

whose solution is $y(t)=2 t^{2}+t$.

The numerical results with $n=20$ are shown in Fig. 4(a), and maximum errors are shown in Fig. 4(b). The former implies that the proposed method gives the classical solution under appropriate settings, and the latter shows that computational errors decay exponentially with respect to $n$.

Example 6. We consider an initial value problem

$$
\begin{aligned}
& t^{3} y^{\prime \prime}(t)+t y^{\prime}(t)=-25 t^{3} \sin (5 t)+5 t \cos (5 t) \quad(-1<t \leq 1) \\
& y(-1)=\sin (5), \quad y^{\prime}(-1)=5 \cos (5)
\end{aligned}
$$




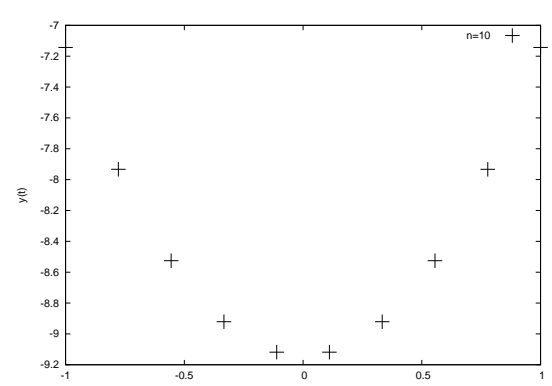

(a) $n=10$

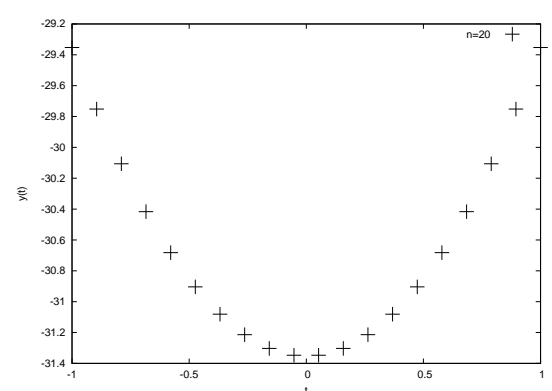

(c) $n=20$

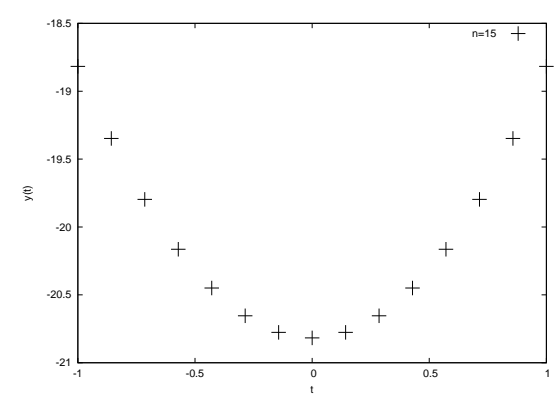

(b) $n=15$

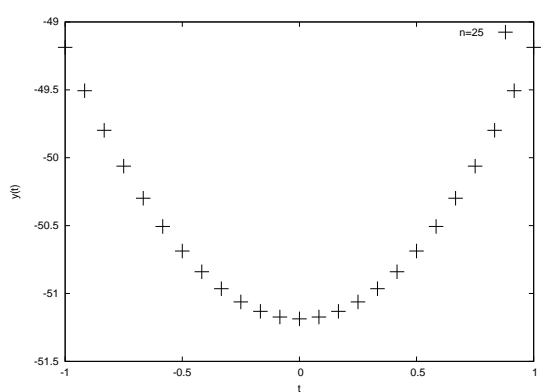

(d) $n=25$

Fig. 3 Numerical Results for Example 4 by 100 Decimal Digits Precision.

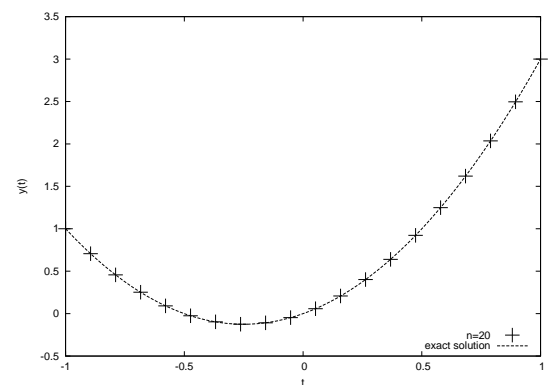

(a) Profile of the Numerical Solution

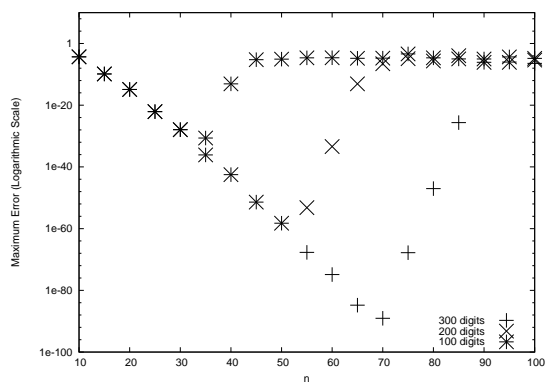

(b) Maximum Error

Fig. 4 Numerical Results for the Boundary Value Problem (Example 5) by Multiple-Precision Arithmetic.

and we set collocation points to $t_{j}=-1+2 j /(n-2), j=1,2, \ldots, n-2$.

Numerical results shown in Fig. 5 have a good coincidence with the exact solution $y(t)=\sin (5 t)$.

Example 7. Let us consider an initial value problem

$$
y^{\prime \prime}(t)=g(t) \quad(-1<t \leq 1), \quad y(-1)=y^{\prime}(-1)=0,
$$




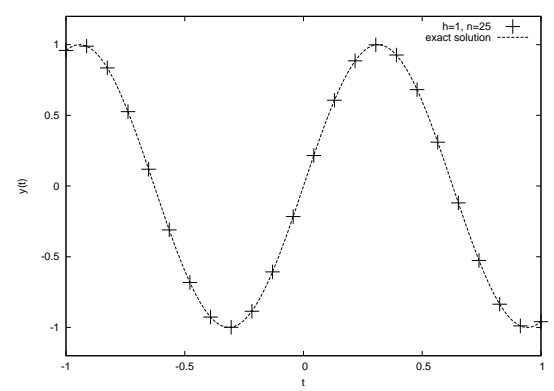

Fig. 5 Numerical Results for the Initial Value Problem (Example 7) for $n=25$ with 100 Decimal Digits. Maximum Error Is Approximately $10^{-13}$.

where

$$
g(t)= \begin{cases}0, & t<0 \\ t, & t \geq 0\end{cases}
$$

For the problem, there exists a unique solution

$$
y(t)= \begin{cases}0, & t<0 \\ \frac{t^{3}}{6}, & t \geq 0\end{cases}
$$

while our numerical results shown in Fig. 6 do not approximate it. We give a remark that the exact solution is not an analytic function but a $C^{2}$-class function, though the proposed method assumes that the solution belongs to the Paley-Wiener space $W\left(\frac{\pi}{h}\right)$, where $h$ is an approximation parameter. We employ $h=1$ in Fig. 6, and more small $h$ in Fig. 7. These results imply that the space does not have enough approximation ability if the solution is not an analytic function.

This numerical experiment seems to show the need of the application of suitable Sobolev spaces as basic approximate function spaces. Of course, we are, at this moment, interested in the classical solutions for the corresponding differential equations.

\section{Final Discussion}

In our new discretization method we will need the precision in some deep way and huge computer resources. However, these both requirements were prepared by H. Fujiwara already (e.g., recall the case of the Laplace transform). Meanwhile, our method is relying on very general linear problems which may be dealt with as shown, for arbitrary linear partial differential equations with very general domains. Furthermore, boundary values and initial values can also be given with wide 


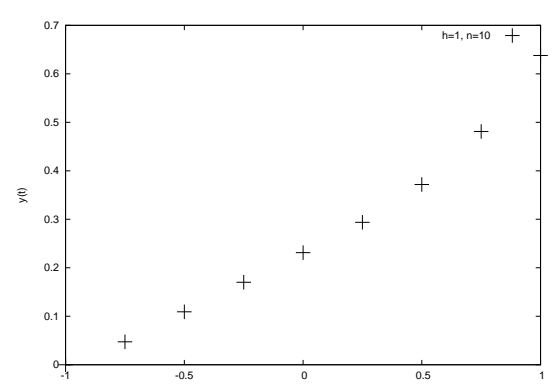

(a) $n=10$

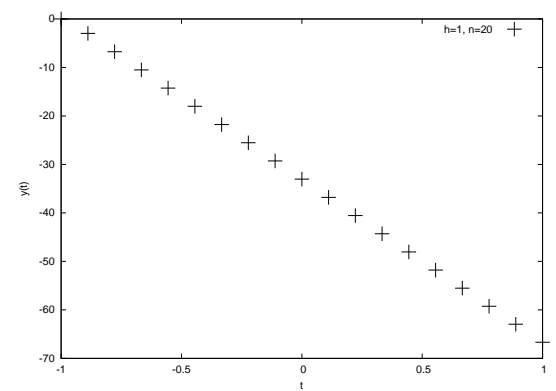

(c) $n=20$

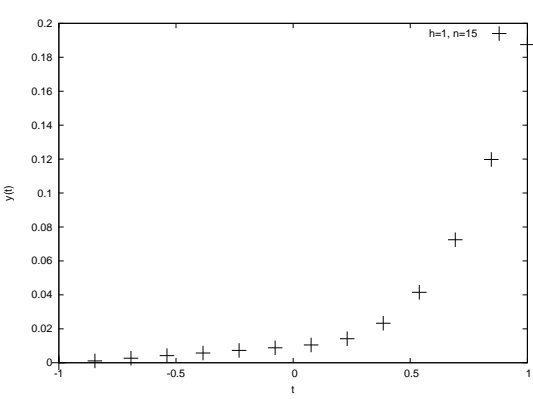

(b) $n=15$

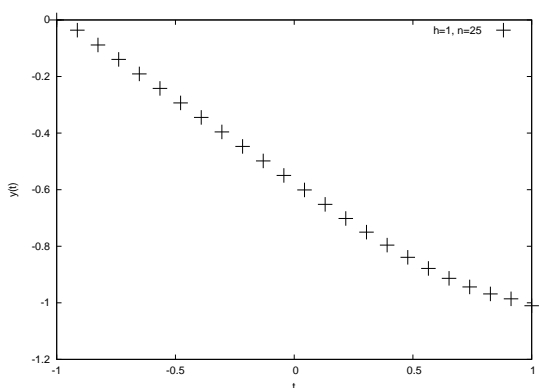

(d) $n=25$

Fig. 6 Numerical Results for Example 7 by 500 Decimal Digits Precision, $h=1$.

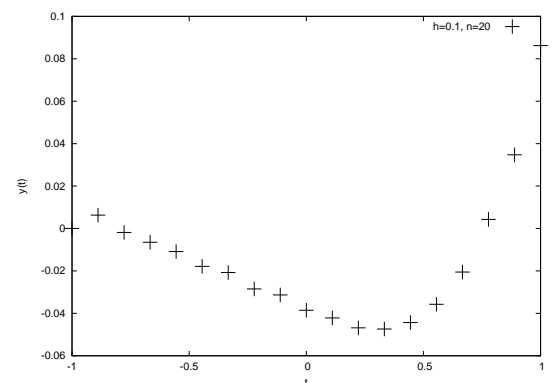

(a) $h=0.1$

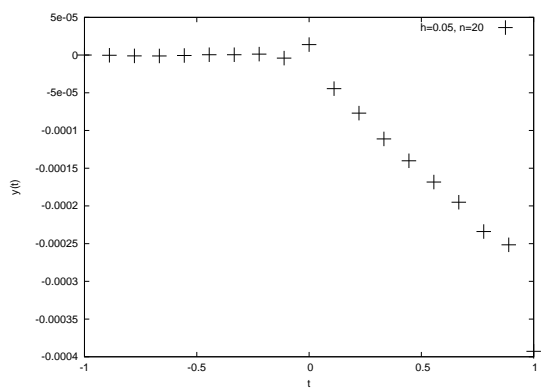

(b) $h=0.05$

Fig. 7 Numerical Results for Example 7 by 500 Decimal Digits Precision, $n=20$.

freedom. Of course, the essential new restriction of the method is that it can be considered for only a finite number of the points of time and space for the equations.

We are looking for some optimal solutions satisfying the differential equations at the given discrete points and so, we are free from important restrictions on the domains which occur on ordinary methods. For instance, this is not the case of the Finite Element Method and the Difference Method which are depending seriously on the domains. In our case, we can consider the problems on any domains. Further- 
more, we can consider the error estimates for the true solutions and our approximate solutions over the global space and time spaces depending on the numbers of the points. However, these error estimates are depending on the specific situation, case by case. Here, the error estimates mean that our approximate solutions of the differential equations satisfying the equations for some discrete number of points and the solutions of the equations are near in some sense. For the unidimensional case we will be easily able to image its meaning. However, on some higher-dimensional cases, its detail and exact meaning are delicate.

Anyhow, error estimates for our approximate solutions are entirely new open problems.

Finally, as a lateral mathematical consequence, we would like to point out that these problems will generate new interesting inequalities.

Acknowledgements This work was supported in part by FEDER funds through COMPETEOperational Programme Factors of Competitiveness ("Programa Operacional Factores de Competitividade") and by Portuguese funds through the Center for Research and Development in Mathematics and Applications and the Portuguese Foundation for Science and Technology ("FCTFundação para a Ciência e a Tecnologia"), within project PEst-C/MAT/UI4106/2011 with COMPETE number FCOMP-01-0124-FEDER-022690. The second author is supported by Grant-in-Aid for Young Scientists (B) (No.23740075). The fourth author is supported in part by the Grant-in-Aid for the Scientific Research (C)(2)(No. 24540113).

\section{References}

1. K. Amano, S. Saitoh, and M. Yamamoto, Error estimates of the real inversion formulas of the Laplace transform, Integral Transform. Spec. Funct. 10 (2000), no. 3-4, 165-178.

2. M. Asaduzzaman and S. Saitoh, Inverses of a family of matrices and generalizations of Pythagorean theorem, Panamer. Math. J. 13 (2003), no. 4, 45-53.

3. J. Benedetto, The Laplace transform of generalized functions, Canad. J. Math. 18 (1966), 357-374.

4. R.P. Boas, Inversion of a generalized Laplace integral, Proc. Nat. Acad. Sci. U. S. A. 28 (1942), 21-24.

5. A.R. Collar, On the reciprocation of certain matrices. Proc. Royal Soc. Edinburgh 59 (1939), 195-206.

6. L.P. Castro, E.M. Rojas, and S. Saitoh, Inversion from different kinds of information and real inversion formulas of the Laplace transform from a finite number of data, Math. Eng. Sci. Aerosp. MESA 1 (2010), no. 2, 181-190.

7. L.P. Castro, H. Fujiwara, S. Saitoh, Y. Sawano, A. Yamada and M.Yamada, Fundamental error estimates inequalities for the Tikhonov regularization using reproducing kernels, International Series of Numerical Mathematics 161, Inequalities and Appications 2010, Springer, Basel 2012, 87-101.

8. L.P. Castro, S. Saitoh, Fractional functions and their representations, Complex Analysis and Operator Theory, to appear, 1-15, DOI: 10.1007/s11785-011-0154-1.

9. L.P. Castro, S. Saitoh, Y. Sawano and A.S. Silva, Discrete linear differential equations, Analysis 32 (2012), 181-198.

10. L.P. Castro and S. Saitoh, Optimal and Approximate Solutions of Singular Integral Equations by means of Reproducing Kernels, Complex Analysis and Operator Theory, to appear.

11. L.P. Castro, H. Fujiwara, M.M. Rodrigues, and S. Saitoh, A new discretization method by means of reproducing kernels, Proceedings of the 20th International Conference on Finite 
or Infinite Dimensional Complex Analysis and Applications, July 29 - August 3, 2012, Hanoi University of Science and Technology, Hanoi, Vietnam, to appear.

12. L.P. Castro, S. Saitoh, Y. Sawano and N.M. Tuan, Approximate solutions of arbitrary linear ordinary differential equations, submitted for publication.

13. L.P. Castro, S. Saitoh, Y. Sawano and A. Yamada, Real inversion of the Laplace transform by a Moore-Penrose generalized inverse construction (manuscript).

14. A.M. Cohen, Numerical Methods for Laplace Transform Inversion, Numerical Methods and Algorithms 5, Springer, New York, 2007.

15. M.D. Choi, Tricks or treats with the Hilbert matrix, Amer. Math. Month. 90 (1983), 301-312.

16. B. Cotterell and J.R. Rice, Slightly curved or kinked cracks, International Journal of Fracture 16 (1980), 155-169.

17. H. Fujiwara, Applications of reproducing kernel spaces to real inversions of the Laplace transform, RIMS Koukyuuroku 1618 (2008), 188-209.

18. H. Fujiwara, T. Matsuura, S. Saitoh, and Y. Sawano, Numerical real inversion of the Laplace transform by using a high-accuracy numerical method, Further Progress in Analysis, 574583, World Sci. Publ., Hackensack, NJ, 2009.

19. H. Fujiwara, Numerical real inversion of the Laplace transform by reproducing kernel and multiple-precision arithmetric, Progress in Analysis and its Applications, 289-295, Proceedings of the 7th International ISAAC Congress, World Scientific, 2010.

20. B.J. González and E.R. Negrin, A distributional inversion formula for a generalization of the Stieltjes and Poisson transforms, Integral Transform. Spec. Funct. 20 (2009), no. 12, 897-903.

21. I.S. Gradshteyn and I.M. Ryzhik, Table of Integrals Series, and Products, Academic Press, New York, 1980.

22. J.R. Higgins, Sampling Theory in Fourier and Signal Analysis: Foundations, Clarendon Press, Oxford, 1996.

23. S.W. Indratno and A.G. Ramm, Inversion of the Laplace transform from the real axis using an adaptive iterative method, Int. J. Math. Math. Sci. 2009, Art. ID 898195, 38 pp., doi:10.1155/2009/898195

24. V.V. Kryzhniy, Regularized inversion of integral transformations of Mellin convolution type, Inverse Problems 19 (2003), 1227-1240.

25. V.V. Kryzhniy, Numerical inversion of the Laplace transform: analysis via regularized analytic continuation, Inverse Problems 22 (2006), 579-597.

26. R.P. Manandhar and L. Debnath, Representation of functions as the Post-Widder inversion operator of generalized functions, Internat. J. Math. Math. Sci. 7 (1984), no. 2, 371-396.

27. N.I. Muskhelishvili, Singular Integral Equations, Noordhoff, Groningen, 1972.

28. F. Natterer, The Mathematics of Computerized Tomography, Classics in Applied Mathematics 32, Society for Industrial and Applied Mathematics (SIAM), Philadelphia, PA, 2001.

29. J. Peng and S.-K. Chung, Laplace transforms and renerators of semigroups of operators, Proc. Amer. Math. Soc. 126 (1998), no. 8, 2407-2416.

30. R.S. Phillips, An inversion formula for Laplace transforms and semi-groups of linear operators, Ann. of Math. 59 (1954), 325-356.

31. E.L. Post, Generalized differentiation, Trans. Amer. Math. Soc. 32 (1930), no. 4, 723-781.

32. A.G. Ramm, Multidimensional Inverse Scattering Problems, Monographs and Surveys in Pure and Applied Mathematics 51, Longman Scientific and Technical, UK, 1992.

33. Th.M. Rassias and S. Saitoh, The Pythagorean theorem and linear mappings, PanAmerican Math. J. 12 (2002), 1-10.

34. S. Saitoh, Hilbert spaces induced by Hilbert space valued functions, Proc. Amer. Math. Soc. 89 (1983), 74-78.

35. S. Saitoh, Integral Transforms, Reproducing Kernels and their Applications, Pitman Res. Notes in Math. Series 369, Addison Wesley Longman, Harlow, 1997.

36. S. Saitoh, N. Hayashi and M. Yamamoto (eds.), Analytic Extension Formulas and their Applications, Kluwer Academic Publishers, Dordrecht, 2001.

37. S. Saitoh, V.K. Tuan and M. Yamamoto, Conditional stability of a real inverse formula for the Laplace transform, Z. Anal. Anwendungen 20 (2001), no. 1, 193-202. 
38. S. Saitoh, Approximate real inversion formulas of the Laplace transform, Far East J. Math. Sci. 11 (2003), 53-64.

39. S. Saitoh, Inverses of a family of bounded linear operators, generalized Pythagorean theorems and reproducing kernels, Ill-posed and non-classical problems of mathematical physics and analysis, 125-141, Inverse Ill-posed Probl. Ser., VSP, Utrecht, 2003.

40. S. Saitoh, Theory of reproducing kernels: Applications to approximate solutions of bounded linear operator functions on Hilbert spaces, Amer. Math. Soc. Transl. Ser. 2, 230, Amer. Math. Soc., Providence, RI, 2010.

41. Y. Sawano, H. Fujiwara and S. Saitoh, Real inversion formulas of the Laplace transform on weighted function spaces, Complex Anal. Oper. Theory 2 (2008), 511-521.

42. F. Stenger, Numerical Methods Based on Sinc and Analytic Functions, Springer Series in Computational Mathematics 20, NY, 1993.

43. V.K. Tuan and D.T. Duc, A new real inversion formula of the Laplace transform and its convergence rate, Fract. Calc. Appl. Anal. 5 (2002), no. 4, 387-394.

44. V.K. Tuan and N.T. Hong, Interpolation Formula in Hardy space, Integral Transforms and Special Function, to appear.

45. S. Yakubovich, A real inversion formula for the bilateral Laplace transform (Russian), Izv. Nats. Akad. Nauk Armenii Mat. 40 (2005), no. 3, 67-79; English translation in J. Contemp. Math. Anal. 40 (2005), no. 3, 66-77.

46. D.V. Widder, The Laplace Transform, Princeton Mathematical Series 6, Princeton University Press, Princeton, NJ, 1941. 